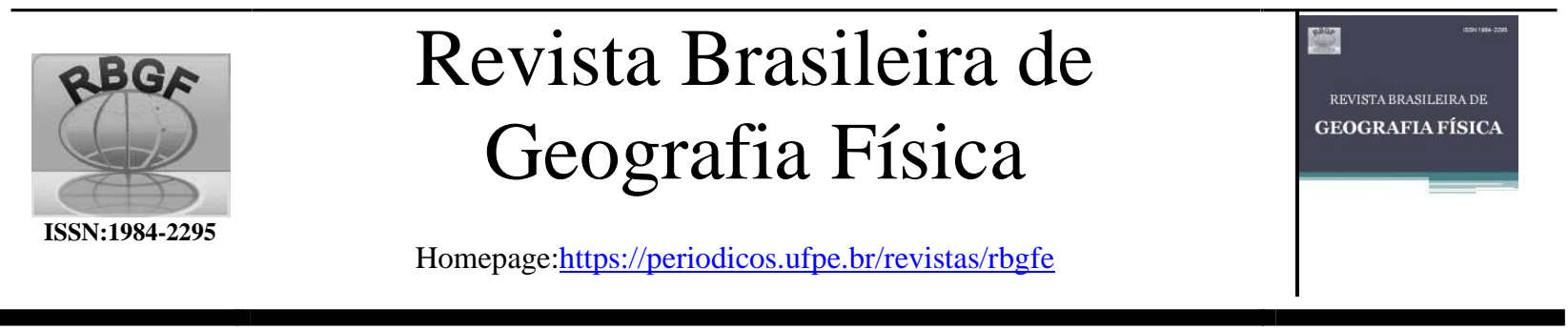

\title{
Análise de tendências anuais e sazonais de extremos da Temperatura da Superfície do Mar próximo à costa da América do Sul no período de 1979 a 2018
}

\author{
Marilia Mitidieri Fernandes de Oliveira ${ }^{1}$, Jorge Luiz Fernandes de Oliveira ${ }^{2}$, Pedro José Farias Fernandes ${ }^{3}$, Nelson \\ Francisco Favilla Ebecken ${ }^{4}$
}

\begin{abstract}
${ }^{1}$ Dr. em Computação de Alto Desempenho, Pesquisadora de Pós-doutorado, Coordenação de Pós Graduação e Pesquisa, Programa de Engenharia Civil, Universidade Federal do Rio de Janeiro, Av. Athos da Silveira Ramos, 149, Centro de Tecnologia, Bloco B, Ilha do Fundão, Caixa Postal 68506, CEP 21941-909, Rio de Janeiro, Rio de Janeiro. (21) 3938-7390 (marilia@ coc.ufrj.br, autor correspondente). ${ }^{2}$ Professor Titular, Departamento de Geografia, Instituto de Geociências, Universidade Federal Fluminense, Av. Gal. Milton Tavares de Souza, s/nº, Campus da Praia Vermelha, Gragoatá, CEP 24210 346, Niterói, Rio de Janeiro. (21) 26295958 (jorgeo@id.uff.br) ${ }^{3}$ Dr. Pesquisador, Laboratório de Geografia Física, Departamento de Geografia, Instituto de Geociências, Universidade Federal Fluminense, Av. Gal. Milton Tavares de Souza, s/nº, Campus da Praia Vermelha, Gragoatá, CEP 24210 346, Niterói, Rio de Janeiro. (21) 26295911 (pj_fernandes@id.uff.br). ${ }^{4}$ Professor Titular, Programa de Engenharia Civil, Universidade Federal do Rio de Janeiro, Av. Athos da Silveira Ramos, 149, Centro de Tecnologia, Bloco B, Ilha do Fundão, CEP 21941-909, Rio de Janeiro. (21) 3938-7390 (nelson@ntt.ufrj.br).
\end{abstract}

Artigo recebido em 16/07/2020 e aceito em 24/11/2020

\section{R E S U M O}

O balanço de calor na interação oceano-atmosfera tem forte influência da temperatura da superfície do mar (TSM) e, portanto, pequenas variações podem levar a variações significativas nos fluxos de calor, os quais desempenham um papel importante nos sistemas climáticos. Excesso de calor armazenado nos oceanos vem causando aumentos na temperatura cujo desequilíbrio de energia torna-se uma das formas de quantificar a taxa de aquecimento global. Estudos recentes mostram que em 2019 ocorreu o maior aquecimento em todo período já registrado. Neste trabalho são investigadas tendências nas series anuais e sazonais da TSM, ao longo da plataforma continental do sul do Brasil, Uruguai e Argentina, utilizando-se seis pontos de grade das reanálises do Era-Interim do European Centre for Medium-Range Weather Forecasts de 1979 a 2018. Testes estatísticos não-paramétricos de Mann-Kendall, declividade Sen e Pettitt foram utilizados para testar a estacionariedade e eventuais mudanças bruscas na média das séries temporais. Foi verificads, também, o número de ocorrência (Noc) de extremos acima do percentil de 90. Resultados da análise descritiva mostram picos nos valores máximos anuais a partir do ano 2000, assim como, tendências positivas nas anomalias e nos Noc de extremos. Os maiores valores de TSM foram verificados no verão (JFM), porém próximo ao sul do Brasil ocorreram, também, em abril e maio de 2018. Testes estatísticos confirmam tendências positivas e mudanças na média entre final de 1990 e início de 2000 , principalmente na TSM Máx. Portanto, concluímos que as análises no padrão de máximos da TSM na região de estudo mostram a ocorrência de um aquecimento no período analisado.

Palavras-chave: Temperatura da superfície do mar, testes estatísticos não-paramétricos, Confluência Brasil-Malvinas

\section{Analysis of annual and seasonal trends in Sea Surface Temperature near the coast of South America from 1979 to 2018}

\begin{abstract}
A B S T R A C T
The heat balance in the ocean-atmosphere interaction has a strong influence on the sea surface temperature (SST) and, therefore, small variations can lead to large variations in heat flows, which play an important role in climatic systems. Excess heat stored in the oceans has been causing temperature increases which energy imbalance becomes one of the ways to quantify the rate of global warming. Recent studies show that in 2019 occurred the biggest warming in the whole period ever recorded. In this research are investigated trends in the SST annual and seasonal series along the continental shelf of southern Brazil, Uruguay and Argentina, using six grid points from Era-Interim reanalysis database from the European Center for Medium-Range Weather Forecasts from 1979 to 2018. Non-parametric statistical tests of Mann-Kendall, Sen Estimator and Pettitt were used to verify the stationarity and eventual abrupt changes in the mean of the series. It was also verified the number of occurrence of extremes above the 90th percentile. Results of descriptive analysis show peaks in the
\end{abstract}


maximum annual values from the year 2000, as well as, positive trends in anomalies and number of extremes. Highest SST values were verified in the summer (JFM) however, in points close to the south of Brazil, they also occurred in April and May 2018. Statistical tests confirm positive trends and change in the mean from the end of the 1990s and early 2000, mainly in the series of maximum SST. Therefore, we conclude that in the study region occurred a warming in the pattern of maximum SST in the analyzed period.

Keywords: sea surface temperature, non-parametric statistical tests, Brazil-Malvinas confluence.

\section{Introdução}

As análises da variação do clima da Terra, nos últimos milhares de anos, a partir de testemunhos em geleiras têm mostrado que a variabilidade climática e os desastres naturais ocorreram em grandes escalas temporais e espaciais. Porém, nos últimos 150 anos, os registros de temperatura começaram a subir mais rapidamente do que ocorria em épocas passadas (Nobre et al., 2012), levando a severas mudanças, as quais vêm sendo observadas e registradas em várias regiões do planeta. As atividades humanas, por sua vez, vêm contribuindo para o aumento de gases de efeito estufa, como $\mathrm{CO}_{2}$, o que poderá alterar, significativamente, a vida na Terra (IPCC, 2019).

A ocorrência de eventos extremos tem se tornado cada vez mais frequentes e intensos, principalmente nas grandes cidades, sendo necessária a elaboração de estratégias para o seu enfrentamento, bem como, a atenuação de seus efeitos (Ryan, 2015; Di Giulio et al., 2019).

$\mathrm{O}$ aumento do número de desastres naturais ocorridos no século XX e inicio do século XXI são atribuídos ao aumento da concentração de gases de efeito estufa na atmosfera, contribuindo para a intensificação de sistemas atmosféricos. A partir da década de 1950 houve um aumento na frequência e intensidade dos desastres naturais em escala global. Esses registros, em sua maioria, estão associados às instabilidades atmosféricas, sendo as inundações o fenômeno que mais têm afetado o Brasil (Banco Mundial, 2012a; Banco Mundial, 2012b). Por outro lado, em décadas recentes tem-se observado períodos de secas extremas em vários países com severas consequências em vários setores, os quais podem estar relacionados tanto às anomalias climáticas quanto efeitos antrópicos (Van Loon et al., 2016).

A evolução do clima avaliada durante o período 2011-2015 e descrita no relatório da Organização Meteorológica Mundial (OMM) visa contribuir para uma melhor compreensão das tendências de aquecimento e eventos extremos, ajudando os tomadores de decisão nos programas sobre as mudanças climáticas de forma mais eficaz. Nesse período, as concentrações dos principais gases de efeito estufa aumentaram e atingiram níveis recordes (WMO, 2016). Como consequência dessas mudanças, tem-se ainda o aumento do nível do mar decorrente do derretimento das calotas polares e geleiras, além da extinção de espécies vegetais e animais e da diminuição da produtividade agrícola (Berz et al., 2001; Mcbean, 2004; Easterling et al., 2007a e b).

Na América do Sul tem sido verificada a ocorrência de eventos meteorológicos significativos. A maior taxa de formação de ciclones no hemisfério sul é verificada entre a Península Antártica (PA) e a Região Sul do Brasil (Hoskins e Hodges, 2005), tendo o continente Antártico características climáticas próprias, de extrema relevância para alterações do clima na América do Sul, pois é muito sensível às mudanças globais (Simões, 2011). Young e Ribal (2019) analisaram dados globais de satélite para determinar as tendências da velocidade do vento na região oceânica e da altura das ondas no período de 1985 a 2018 (33 anos). Um extenso banco de dados foi obtido através de altímetros, radiômetros e scatterometer (medidas de dispersão), mostrando pequenos aumentos na média das variáveis e maiores aumentos em condições extremas (percentil de 90), principalmente no oceano Antártico.

Fenômenos atmosféricos transientes que ocorrem em escala sinótica são descritos por Hoskins e Hodges (2005) relacionados à variabilidade oceânica nas Regiões Sul e Sudeste do Brasil, especialmente as regiões costeiras, sendo mais propícias a sofrer os impactos das mudanças climáticas através de um eventual aumento de eventos extremos.

$\mathrm{Na}$ América do Sul, os contrastes térmicos entre as Correntes do Brasil (CB) e Corrente das Malvinas (CM), as quais possuem características físico-químicas próprias contribuem para a formação de fortes gradientes de momentum e calor, gerando fluxos verticais entre as massas d'água na interface oceano-atmosfera que afetam as estruturas termodinâmicas da atmosfera (Pezzi et al., 2005). O aquecimento nessa interface torna-se um fator importante no desenvolvimento de ciclogêneses, onde a CB tem um papel fundamental 
no aumento da intensidade e frequência desses sistemas meteorológicos na região oceânica entre o sul do Brasil e a Argentina (Smith, 2000; Vera et al., 2002; Matano et al., 2010) próximo da foz do rio da Plata, onde gera uma região de alta variabilidade de mesoescala conhecida por Confluência Brasil-Malvinas (CBM). Podestá et al. (1991) verificaram que as amplitudes anuais da TSM tendem a aumentar na direção da plataforma continental e Lentini et al., (2000) identificaram amplitudes significativas latitudinalmente sobre a plataforma, concluindo que essas variações seriam causadas pelo aumento do transporte da $\mathrm{CB}$ durante o verão e da CM durante o inverno.

A TSM tem uma influência preponderante no balanço de calor na interação oceano-atmosfera e, portanto, pequenas variações na TSM podem levar à ocorrência de grandes variações nos fluxos de calor, os quais desempenham um papel relevante nas condições climáticas locais, regionais e globais (Pezzi et al., 2016).

Em escalas de tempo mais longas, poucos estudos caracterizaram a variabilidade da CBM. Zavialov et al. (1999) identificaram variações de longo prazo na TSM, no período de 1854-1994 com tendência de aquecimento secular de $1,2^{\circ} \mathrm{C}$ a $1,6^{\circ} \mathrm{C}$ na plataforma continental, principalmente nas proximidades dos estuários do rio da Prata e lagoas dos Patos-Mirim. Após análise mais detalhada constataram um aquecimento mais significativo após os anos 1940. As análises foram realizadas separadamente para temperaturas no verão e inverno e ambos os casos apresentaram tendência secular positiva.

Diaz et al,. (1998) descreveram o ciclo anual de precipitação e investigaram as relações entre anomalias dessa variável com as anomalias da TSM nos oceanos Pacífico e Atlântico, analisando a precipitação no Uruguai e no Rio Grande do Sul. Os autores verificaram que, quando as anomalias de TSM ocorrem em ambos os oceanos simultaneamente, as anomalias de precipitação podem tanto ser aumentadas como reduzidas.

Haylock et al. (2006) examinaram observações diárias de precipitação para determinar através de índices climáticos as mudanças na precipitação total e extrema no período de 1960 a 2000 desde o Equador, norte do Peru, sul do Brasil, Paraguai, Uruguai e norte e centro da Argentina. A análise entre os índices e as TSM mostrou padrões do tipo ENSO que contribuem para as tendências observadas nos índices de precipitação. Doyle e Barros (2002) destacam a importância da relação entre a TSM no Atlântico Sudoeste, e a Zona de
Convergência do Atlântico Sul (ZCAS), a qual influencia as monções de verão da América do Sul, período geralmente mais chuvoso na maior parte da Região Norte, Central, Sudeste e Sul do continente. Considerando-se as condições biogeoquímicas, as águas da $\mathrm{CM}$ que penetram na plataforma da região da Patagônia influenciam os ecossistemas regionais onde se observa uma quantidade significativa de nutrientes (Piola et al., 2009; Piola et al., 2018). Essa região possui uma atividade biológica bastante elevada, sendo considerado um ecossistema marinho com altas taxas de produtividade (Sabatini et al., 2004). Já a região da plataforma brasileira comparada com a da Patagônia é mais pobre na estrutura da cadeia trófica (Matano et al., 2010). Porém, a relação entre variáveis oceanográficas e o ciclo de vida de peixes, como a sardinha verdadeira (Sardinella brasiliensis), no litoral brasileiro tem sido investigada por ser um dos recursos vivos mais importantes para a pesca no Brasil, localizando-se na região costeira entre os estados do Rio de Janeiro e Santa Catarina (Pezzi et al., 2016). Gigliotti et al. (2010) descreveram sobre a expansão e retração de hábitat para essa espécie, determinando uma relação entre essas ocorrências e oscilações da TSM locais, o que torna essa espécie bastante dependente de processos oceanográficos locais.

Outro aspecto importante no desenvolvimento de sistemas meteoceanográficos devido ao aquecimento refere-se ao aumento da evaporação e, consequentemente, da umidade na atmosfera mais aquecida, levando a ocorrência de eventos extremos com formações de ciclones, chuvas intensas e inundações (Collins et al., 2018).

A zona costeira do litoral Sul-Sudeste brasileiro é bastante vulnerável à ação de ciclones evoluindo ao longo da costa. Esses sistemas induzem a subida do nível do mar na região costeira, resultando em grandes ondas com efeitos danosos no litoral devido ao comportamento extremo do vento ao longo da Região oceânica Sul e Sudeste do Brasil (Oliveira et al., 2011). Rocha et al. (2016), verificam padrões de circulação atmosférica associados aos ciclones no Oceano Attântico Sul, próximo à costa das Regiões Sul e Sudeste do Brasil, utilizando dados da reanálise do ERA-Interim de outubro a abril de 2003 a 2013. Os autores mostram que em média ocorreram 17 casos de ciclones no período, porém em 2009 houve um aumento significativo com 27 ocorrências registradas.

Estudos recentes têm mostrado que a sensibilidade climática varia no tempo conforme a 
ocorrência de troca de calor entre a superfície e os oceanos profundos, fazendo com que evolua o padrão de aquecimento referente à superfície do oceano. Esse efeito no padrão de aquecimento na superfície dos oceanos, o qual apresenta o forçamento radiativo em evolução (gases de efeito estufa vs. aerossóis), contribui para os desafios na estimativa da sensibilidade climática a partir de observações instrumentais (Xie, 2020). Enquanto as concentrações atmosféricas de gases de efeito estufa são quase uniformes na horizontal, o aquecimento da superfície do oceano apresenta variações espaciais. O padrão de aquecimento do oceano afeta a circulação atmosférica e altera padrões de precipitação, bem como no ENOS e na sensibilidade climática global. A distribuição de radiação além de atuar como forçante da circulação oceânica, também é importante na formação do padrão de aquecimento da TSM, sendo necessários mais estudos para entender e quantificar os vários mecanismos atuantes nessa formação (Collins et al., 2018).

O excesso de calor armazenado nos oceanos vem causando aumentos na temperatura do oceano (Abram et al., 2019) cujo desequilíbrio de energia torna-se uma das formas de quantificar a taxa de aquecimento global (Cheng et al., 2019). Em Cheng et al. (2020) observa-se que no ano de 2019, os dados mostram o maior aquecimento em todo período histórico já registrado, especialmente acima dos $2.000 \mathrm{~m}$ de profundidade. Os autores analisaram a seção vertical das tendências lineares da temperatura dos oceanos Atlântico, Pacífico e Índico entre 1960 e 2019 entre a superfície e 2000 $m$ de profundidade, a partir do oceano Antártico. No oceano Atlântico, entre $50^{\circ} \mathrm{S}$ e $30^{\circ} \mathrm{S}$ de latitude, a tendência da TSM é superior a $1,2^{\circ} \mathrm{C} / 100$ anos.

Aumentos na temperatura do oceano reduzem o oxigênio dissolvido o que afeta significativamente a vida marinha, principalmente os corais e demais organismos sensíveis à

\section{Material e Métodos}

Localização e caracterização da área de estudo

A área de estudo situa-se na região oceânica da plataforma continental do sul do Brasil, Uruguai e Argentina entre as latitudes de $43^{\circ} \mathrm{S}$ e $29^{\circ} \mathrm{S}$ e entre temperatura e às propriedades químicas inerentes aos ambientes marinhos (Aristegui et al., 2019).

A TSM é uma variável importante na interação oceano-atmosfera e mudanças climáticas com vários estudos mostrando tendências positivas nas últimas décadas (Diaz et al., 1998; Lentini et al., 2000; Turner et al., 2005; Turner et al., 2016; Bombardi et al., 2017).

$\mathrm{Na}$ última década os estados do Espirito Santo, Rio de Janeiro e São Paulo têm sido afetados por eventos meteorológicos, conhecidos como ciclones subtropicais e extratropicais, os quais vêm se intensificando e causando chuvas intensas com ventos fortes, alagamentos, deslizamentos e queda de centenas de árvores de grande porte. Esses sistemas associados, principalmente, às passagens de frentes frias atuam de forma mais severa em regiões do sul do Brasil que normalmente é mais favorável à ocorrência de eventos extremos. Essas condições meteorológicas anômalas podem estar provavelmente, relacionadas às anomalias positivas da TSM, bem como, outros sistemas meteorológicos de altitude como cavados, os quais são sistemas abertos de baixa pressão atmosférica.

Neste artigo são apresentados os resultados preliminares do projeto de pesquisa de pósdoutorado desenvolvido no Programa de Engenharia Civil (COPPE) da Universidade Federal do Rio de Janeiro que analisa o comportamento de padrões atmosféricos extremos na região do oceano Atlântico Sul desde a Península Antártica (PA) até o Sudeste do Brasil, por ser uma área relevante para a formação de sistemas transientes causadores de desastres ambientais que afetam a maior parte da população, principalmente, nas regiões costeiras (Rocha et al., 2016). Portanto, o objetivo desta pesquisa é analisar as tendências das séries de máximos anuais e sazonais da TSM nos últimos 40 anos ao longo da costa da Região Sudeste do Brasil.

as longitudes de $48^{\circ} \mathrm{W}$ e $61^{\circ} \mathrm{W}$, onde se encontra a posição média da $\mathrm{CBM}$, que é uma zona frontal formada pelo encontro entre a $\mathrm{CB}$ que flui para o sul e a CM que flui para o norte (Figura 1). A dinâmica ao longo da costa leste do continente Sulamericano é caracterizada pelo encontro das águas frias subantárticas e as águas subtropicais mais quentes (Peterson e Stramma, 1991). 


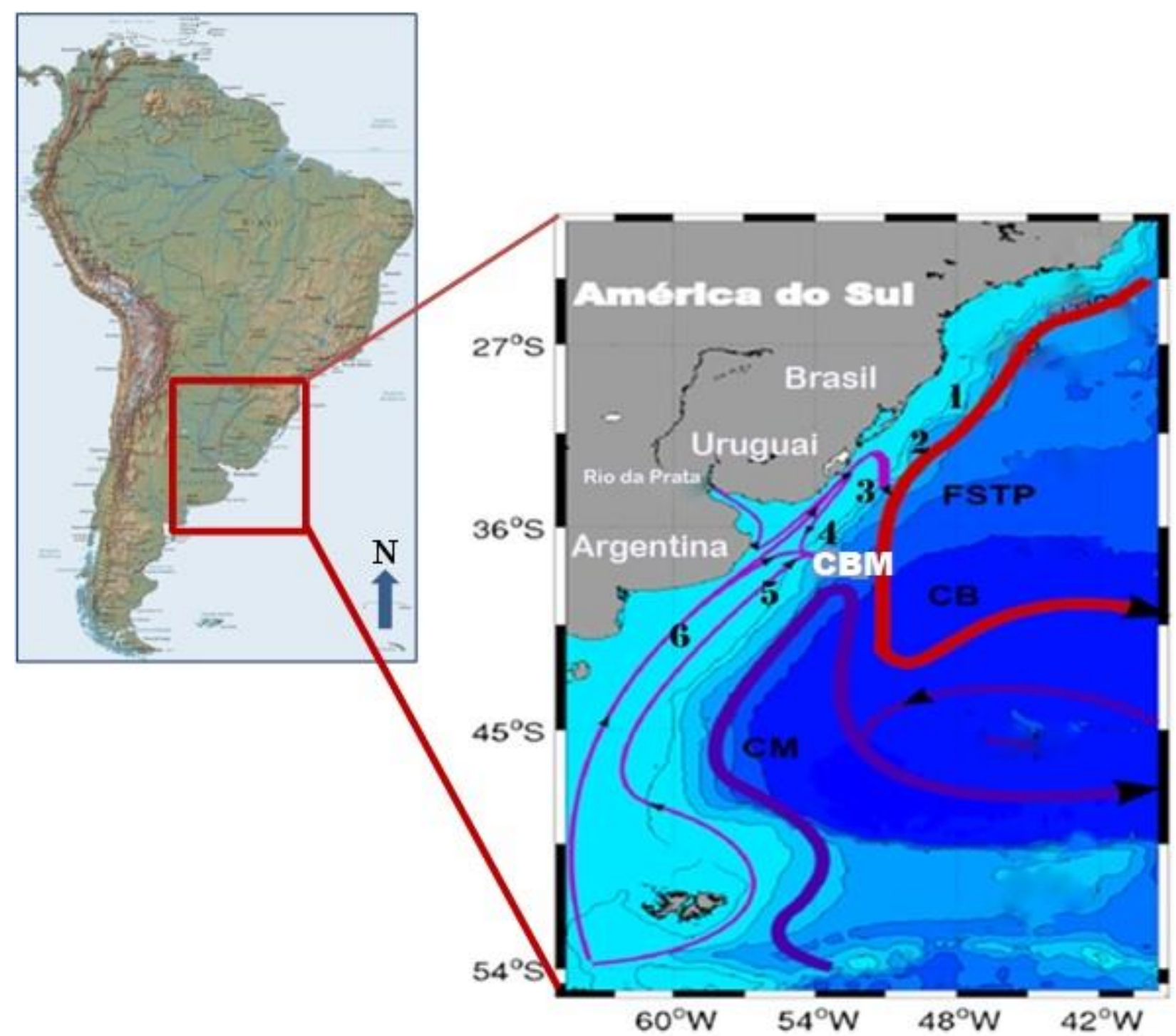

Figura 1. Mapa da área de estudo com destaque da localização dos pontos (preto) referentes aos dados de reanálise do ERA-Interim na área oceânica da região da plataforma continental entre o sul do Brasil e leste da Argentina onde ocorre a CBM. O mapa mostra, também, a circulação de águas superficiais do oceano Atlântico Sul com os contornos da CM (azul) e CB (vermelho) e a Frente Subtropical de Plataforma (FSTP). Fonte: Adaptado de Matano, 2010.

A Tabela 1 mostra a localização dos seis pontos selecionados. $\mathrm{O}$ ponto 4 situa-se entre $\mathrm{o}$ Uruguai e a Argentina, sendo o mais próximo da CBM.

Nessa região encontra-se a borda oeste do giro subtropical do Atlântico Sul, a qual é paralela ao continente Sul-americano, delimitada pela CB, sendo uma corrente de contorno originada na bifurcação da Corrente Equatorial (CE), em torno de $10^{\circ} \mathrm{S}$ (Figura 2).
Essa corrente flui para o sul ao longo da plataforma continental até alcançar a região onde se encontra a CBM, ou seja, a formação da CBM é caracterizada pela presença do giro da Água Central do Atlântico Sul (ACAS) que se mistura à Água Tropical transportada pela CB e a Água Subantártica transportada pela CM, as quais fazem parte da circulação superficial do oceano Attântico Sudoeste (Soppa et al., 2011). 
Tabela 1. Localização dos pontos de grade selecionados da base de dados do ERA-Interim para análise da TSM no sudoeste do oceano Atlântico Sul entre o sul do Brasil e leste da Argentina.

\begin{tabular}{lccc}
\hline \multicolumn{1}{c}{ Pontos } & Latitude & Longitude & Período \\
\hline 6 (Argentina) & -42.80 & -60.38 & $1980-2018$ \\
5 (Argentina) & -39.14 & -56.54 & $1979-2018$ \\
4 (Foz do Prata) & -36.07 & -53.79 & $1979-2018$ \\
3 (Uruguai) & -34.22 & -51.85 & $1979-2018$ \\
2 (R. G. Sul) & -32.30 & -50.50 & $1979-2018$ \\
1 (R. G. Sul) & -29.37 & -48.05 & $1979-2018$ \\
\hline
\end{tabular}

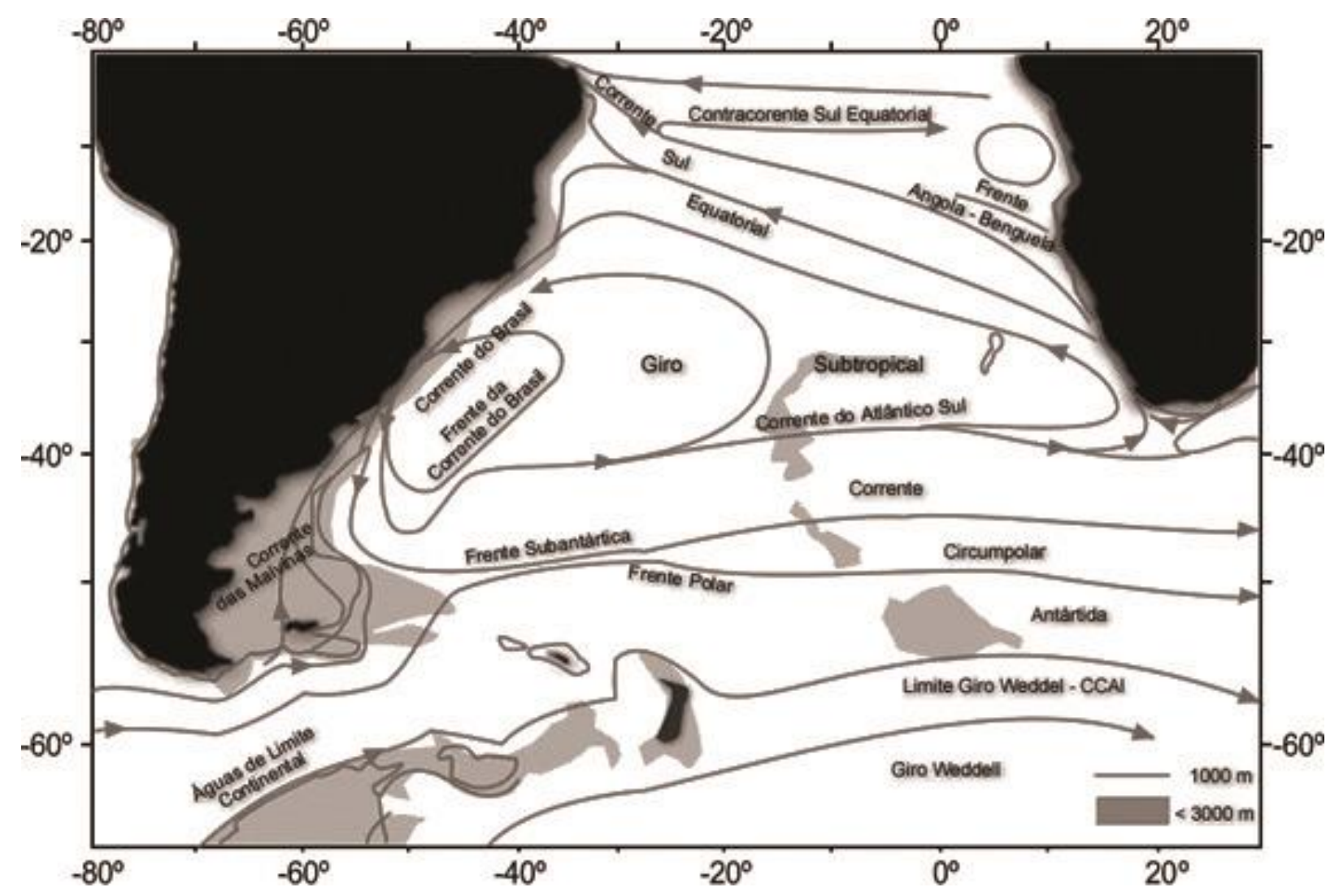

Fonte: Adaptada de Peterson e Stramma (1991).

Figura 2. Esquema de larga escala das correntes superficiais e frentes do oceano Atlântico Sul.

A CBM é caracterizada, em parte, pelos principais aspectos físicos, tanto da $\mathrm{CB}$ referentes ao transporte de águas quentes e salinas quanto da CM no transporte de águas frias e menos salinas, interagindo com processos atmosféricos como fluxos de calor, estabilidade da camada limite atmosférica e ventos, importantes processos ligados ao acoplamento oceano-atmosfera nessa região (Lumpkin e Garzoli, 2011; Pezzi et al., 2016; Freitas et al., 2019). A latitude de separação da costa da CB caracteriza-se por uma variabilidade sazonal que se estende mais ao sul durante o verão e mais ao norte durante o inverno com relação à latitude média, em torno de $36^{\circ} \mathrm{S}$. A CB continua fluindo em direção ao sul entre os paralelos $40^{\circ} \mathrm{S}$ e $45^{\circ} \mathrm{S}$ após sua separação da costa, dividindo-se em dois ramos, um que flui para norte e outro que continua para sul com o fluxo de retorno da CM (Olson et al., 1988),

A Corrente Circumpolar Antártica (CCA) favorece trocas e teleconexões entre as bacias oceânicas, influenciando climas regionais nos lugares mais remotos. O principal mecanismo controlador da CCA é o forte sistema atmosférico de ventos de oeste que predomina em toda a extensão do oceano Austral de $40^{\circ} \mathrm{S}$ a $65^{\circ} \mathrm{S}$. Acredita-se que a variabilidade espaço-temporal desse sistema é controlada, primeiramente, pelo

Oliveira, M. M. F., Oliveira, J. L. F., Fernandes, P. J. F., Ebecken, N. F. F. 
Southern Annular Mode (SAM), conhecido como Oscilação Antártica (OA), o qual descreve o movimento norte-sul de ventos de oeste predominantes das latitudes médias para altas (Mata e Garcia, 2011). A CM è formada a partir de um ramo da CCA que gira com movimento anticiclônico após fluir para norte a partir do Estreito de Drake (Lentini et al., 2002). A CM acompanha a região da quebra da plataforma continental até aproximadamente $38^{\circ} \mathrm{S}$, onde também se separa da costa e segue na direção leste da bacia Atlântica. Na região da confluência, em torno dessa latitude, onde as duas correntes se encontram e fluem para leste, ocorre uma separação de ambas em duas frentes, uma subtropical e outra subantártica, marcando o limite sul do giro subtropical descrito acima, local onde se observa a ocorrência de convergência entre as duas correntes de contorno associada à geração de meandros e intensa vorticidade (Stramma e Peterson, 1990).

\section{Aquisição das Reanálises do ERA-Interim}

$\mathrm{Na}$ presente pesquisa foram utilizados dados de reanálises do ERA-Interim e baixados a partir de uma janela de $20^{\circ} \mathrm{S}$ a $70^{\circ} \mathrm{S}$ de latitude e de $20^{\circ} \mathrm{W}$ a $70^{\circ} \mathrm{W}$ de longitude. Para baixar a base de dados foi desenvolvido um programa na linguagem Python para o período de janeiro de 1979 a dezembro de 2018 (40 anos) nos horários de 00, 06, 12 e 18 UTC referentes à pressão atmosférica ao nível médio do mar, componentes zonal (u) e meridional (v) do vento, temperatura do ar a 2 metros do solo e temperatura da superfície do mar.

\section{Métodos Estatísticos de Tendência}

Existem vários métodos estatísticos para testar a estacionariedade de séries temporais climatológicas. Uma série temporal é considerada estacionária quando apresenta comportamento aleatoriamente homogêneo no tempo em relação a uma média constante, porém a maioria das séries apresenta algum tipo de não estacionariedade, ou seja, tendência. Séries temporais não homogêneas podem contribuir para interpretar erroneamente eventos extremos e ser enganosas na interpretação de tendências que eventualmente ocorram (Kocsis et al., 2020). Dentre os vários testes estatísticos, encontram-se os classificados em paramétricos e não paramétricos. Os métodos paramétricos (regressão linear) assumem que os dados sejam independentes e, em geral, normalmente distribuídos, podendo ser ajustados a uma distribuição teórica paramétrica, enquanto que os
Os pontos foram escolhidos dentro dessa janela, considerando-se as áreas de formações de sistemas meteoceanográficos transientes na região em torno da CBM entre $43^{\circ} \mathrm{S}$ e $29^{\circ} \mathrm{S}$ de latitude e $48^{\circ} \mathrm{W}$ e $61^{\circ} \mathrm{W}$ de longitude (Tabela 1). O ERA-Interim é uma reanálise da atmosfera global que abrange o período de dados desde 1979 (originalmente, o ERA-Interim decorreu de 1989, mas a extensão de 10 anos de 1979-1988 foi produzida em 2011) e continua em tempo real. À medida que a base de dados do ERA-Interim avança no tempo, as atualizações do arquivo são realizadas mensalmente. Foi desenvolvido um modelo atmosférico acoplado a um modelo de ondas oceânicas com uma grade de $1,0^{\circ}$ x $1,0^{\circ}$ de latitude/longitude. Uma documentação abrangente do sistema de reanálise da ERA-Interim pode ser verificada em Berrisford et al. (2011) e Dee et al. (2011), este último como um artigo de acesso aberto no revista Trimestral da Royal Meteorological Society.

Primeiramente os valores da TSM anual e sazonal para o período de 1979 a 2018 foram utilizados para identificar tendências monotônicas nos seis pontos distribuídos ao longo da plataforma continental entre os limites latitudinais. Como os dados são em intervalos de 6 em 6 horas (quatro informações por dia), cada série temporal referente a cada ponto contém em média 1460 dados. Desse conjunto de dados foram extraídos os valores máximos anuais, gerando uma série temporal de 40 dados respectivos ao período de análise, bem como séries sazonais para o período de interesse (janeiro, fevereiro e março).

não paramétricos não fazem distinção quanto à distribuição a que os dados pertençam, exigindo apenas a independência entre os dados da amostra (Alexandre et al., 2010; Blain, 2011). A aplicação do teste de tendência não paramétrico de MannKendall (Mann, 1945; Kendall, 1975) é recomendada pela OMM para detectar tendências monotônicas estatisticamente significativas de longo prazo em conjuntos de dados ambientais (Irannezhad et al., 2016; Kocsis et al., 2020). O uso do teste de MK é simples e robusto em relação aos desvios de normalidade e não estacionariedade, e pode lidar com valores ausentes abaixo do limite de detecção (Yue et al., 2002; Gavrilov et al., 2016).

As mudanças bruscas na média são um dos fatores que apontam para a falta de homogeneidade nos dados de séries temporais (Killick et al., 2012; Rahman et al., 2017) e para detectar essas mudanças, os testes mais usados são os não paramétricos de Mann-Kendall (MK), Spearman's 
rho (1904) e o teste de Pettitt (1979) que detecta tanto a falta de homogeneidade quanto o ponto de

Uma versão do teste de Mann-Whitney utiliza o teste de Pettitt com o objetivo de verificar se duas amostras $\left(\mathrm{X}_{1}, \ldots . \mathrm{X}_{\mathrm{t}}\right.$ e $\left.\mathrm{X}_{\mathrm{t}+1}, \ldots . . \mathrm{X}_{\mathrm{T}}\right)$ são da mesma população. Este teste localiza o ponto onde ocorreu uma mudança brusca na média da série dentro de uma significância estatística calculada a partir de um teste de hipótese (Back, 2001; Liu et al. 2012). Nessa pesquisa, uma abordagem nãoparamétrica do teste de Pettitt foi escolhida, o qual ainda é amplamente utilizado para detectar a não homogeneidade nas séries temporais e consequentemente identificar o ponto onde a mudança ocorre (Kocsis et al., 2020). Esse teste considera a hipótese nula como dados homogêneos, contra a hipótese alternativa de que há um dado no qual há uma alteração. Esse teste foi realizado a um nível de significância de 5\%.

Teste de Tendência Mann-Kendall, estimador Sen e Teste de Pettitt

$\mathrm{O}$ teste de tendência monotônica de MK está intimamente relacionado com o coeficiente de correlação de Kendall. De acordo com o teste MK, duas hipóteses são testadas: a hipótese nula $\left(\mathrm{H}_{0}\right)$, em que não há tendência na série temporal e a hipótese alternativa $\left(\mathrm{H}_{\mathrm{a}}\right)$, que existe uma tendência significativa na série para um dado nível de significância $p$. Se o valor calculado $p$ for menor que o nível escolhido $\alpha$ (por exemplo, $\alpha=5 \%$ ), $\mathrm{H}_{0}$ (não há tendência) deve ser rejeitada e $\mathrm{H}_{\mathrm{a}}$ (há tendência significativa) deve ser aceita. Caso $p$ seja maior que o nível de significância $\alpha, \mathrm{H}_{0}$ não pode ser rejeitada. A estatística do teste de MK é apresentada na equação 1 para uma série de dados $\mathrm{X}=\left(\mathrm{X}_{1}, \mathrm{X}_{2}, \mathrm{X}_{3}, \ldots \ldots \ldots . . . \mathrm{X}_{\mathrm{n}}\right)$ proveniente de uma amostra de $\boldsymbol{n}$ variáveis aleatórias independentes e identicamente distribuídas (iid):

$S=\sum_{k=1}^{n-1} \sum_{j=k * 1}^{n} \operatorname{sinal}\left(x_{j}-x_{k}\right)$

onde $\mathrm{j}>\mathrm{ke}$

$\mathrm{k}=1,2, \ldots . ., \mathrm{n}-1$

$\mathrm{j}=2,3, \ldots \ldots ., \mathrm{n}$

O nível de significância $\boldsymbol{\alpha}$ adotado é de $\boldsymbol{\alpha}=$ $0,05=5 \%$ para o teste de MK. Se a probabilidade $p$ do teste for menor que o nível $\boldsymbol{\alpha}(p<\boldsymbol{\alpha})$, uma

No teste de MK o resultado da tendência analisada é mostrado através do escore Tau de Kendall indicando diminuição ou aumento nos mudança em uma série temporal (Arikan e Kahya, 2019).

n é o número de dados;

o sinal $\left(\mathrm{x}_{\mathrm{j}}-\mathrm{x}_{\mathrm{k}}\right)$ é calculado pela equação 2 :

$\operatorname{sinal}\left(x_{j}-x_{k}\right)\left\{\begin{array}{r}1 \text { se } x_{j}-x_{k}>0 \\ 0 \text { se } x_{j}-x_{k}=0 \\ -1 \text { se } x_{j}-x_{k}<0\end{array}\right.$

Segundo Kocsis et al. (2020), Kendall (1975) provou que $S$ é assintoticamente normalmente distribuído com média $E(S)=0$ e variância $\operatorname{Var}(\mathrm{S})$, conforme a equação 3 para valores sem elementos vinculados, ou seja, valores iguais:

$\operatorname{Var}(S)=\left[\frac{n(n-1)(2 n+5)}{18}\right.$

Se houver elementos vinculados, a variância é calculada de acordo com a equação 4 :

$\operatorname{Var}(S)$

$=\frac{\left\{n(n-1)(2 n+5)-\sum_{p-1}^{g} t_{p}\left(t_{p}-1\right)\left(2 t_{p}+5\right\}\right.}{18}$

onde:

g é o número de grupos vinculados ao conjunto de dados;

$\mathrm{t}_{\mathrm{p}}$ é o número de dados no $\mathrm{p}^{\text {th }}$ grupo;

n é o número de dados da série.

Desse modo, a variável randômica $\mathrm{Z}$ é usada no teste de hipótese conforme a equação 5 :

$Z(M K)=\left\{\begin{array}{l}\frac{s-1}{\sqrt{\operatorname{Var}(S)}} \text { se } S>0 \\ 0 \text { se } S=0 \\ \frac{s+1}{\sqrt{\operatorname{Var}(S)}} \text { se } S<0\end{array}\right.$

onde:

a) valor positivo de $Z$ indica tendência ascendente;

b) valor negativo de $\mathrm{Z}$ indica tendência descendente; $\mathrm{e}$

c) para $Z=0$ não há tendência.

tendência estatisticamente significante existe, enquanto, para um valor de $p>\alpha$, existe uma tendência insignificante.

atributos da série quando for negativo ou positivo, respectivamente. Além disso, a tendência apontada por esta metodologia é considerada significativa 
quando p-valor apresentar um valor menor que $\alpha=$ 0,05 . O teste de MK apesar de eficiente e robusto não fornece os valores das tendências detectadas, portanto, pode ser complementado pelo estimador de inclinação da tendência proposto por Sen (1968) que consiste em um método não paramétrico que calcula a alteração por unidade de tempo. $\mathrm{O}$ método

onde $\mathrm{i}<\mathrm{j}$ e $\mathrm{X}_{\mathrm{i}}$ e $\mathrm{X}_{\mathrm{j}}$ representam os dados da série nos anos i e j. $\mathrm{O}$ valor positivo (negativo) para $\mathrm{Q}$ indica tendência crescente (decrescente). Se existem $\mathrm{N}$ valores da série analisada para cada par $\mathrm{j}$

O estimador de declive de Sen é a mediana dos N valores de Qij. (Sen 1968). Este é um parâmetro do método que pode calcular a alteração por unidade de tempo. Os $\mathrm{N}$ valores de $\mathrm{Q}$ são classificados dos menores para os maiores e a mediana dos valores $\mathrm{N}$ de $\mathrm{Q}$ fornece a inclinação da tendência (estimador de Sen). O valor obtido com o estimador é multiplicado pelo período total dos dados. Com isso, tem-se uma estimativa de quanto a variável está se alterando no decorrer do tempo. A inclinação de Sen foi estimada em um nível de confiança de 95\%. O teste de Pettitt (MannWhitney) admite como hipótese nula $\left(\mathrm{H}_{0}\right)$ ausência de ponto de mudança na série e hipótese alternativa $\left(\mathrm{H}_{1}\right)$ de que existe ponto de mudança A estatística não-paramétrica do teste de Pettitt $\left(\mathrm{U}_{\mathrm{t}, \mathrm{n}}\right)$ faz uma contagem do número de vezes que um dado da primeira amostra é maior do que um dado da segunda amostragem (Back,2001; Santos et al, 2016; Moreira e Naghettini, 2016), podendo ser escrita na forma das equações 8 e 9:

$U_{t, T}=\sum_{i=1}^{t} \sum_{j=t+1}^{T} \operatorname{sinal}\left(X_{i}-X_{j}\right)$

onde:

$\operatorname{sinal}(x)=\left\{\begin{array}{r}1 \text { se } x>0 \\ 0 \text { se } x=0 \\ -1 \text { se } x<0\end{array}\right.$

Assim, tem-se que a estatística $U t, T$ é calculada para os valores de $1 \leq \mathrm{t} \leq \mathrm{T}$, e o máximo valor absoluto de $U t, T$ é definido pela estatística $\mathrm{k}_{\mathrm{T}}$ do teste. Essa estatística localiza o ponto em que

\section{Resultados e discussão}

\section{Estatística Descritiva das Séries de TSM}

As análises das estatísticas descritivas dos dados anuais da TSM são apresentadas nas Tabelas 2, 3 e 4 referente aos valores máximos, mínimos, médias, desvio padrão (DP), coeficiente de variação de Sen usa um modelo linear para estimar a inclinação e a variação dos resíduos que deve ser constante no tempo (Tao et al., 2014; da Silva et al., 2015) estimado através da equação 6 :

$Q_{i j}=\frac{X_{j}-X_{i}}{j-i}$

> i, então o número de pares $(\mathrm{N})$ estimados de $\mathrm{Q}$ é dado pela equação 7 :

$N=\frac{n(n-2)}{2}$

ocorreu uma mudança brusca na média de uma série, caracterizando a falta de homogeneidade nos dados. A significância do teste é obtida através da equação 10:

$P \cong 2 \exp \left(\frac{-6 k_{T}^{2}}{T^{3}+T^{2}}\right)$

onde:

P é o nível de significância $(p<0,05)$;

$\mathrm{K}_{\mathrm{T}}$ é o valor crítico;

$\mathrm{T}$ é o $\mathrm{n}^{\circ}$ de anos da série.

A hipótese nula é rejeitada quando $P$ é menor do que o nível de significância $(0,05)$. Os dados da TSM utilizados nesta pesquisa foram processados com programas em linguagem Python e editados na forma de séries temporais, utilizando o software Excel. Primeiramente, foi realizada a estatística descritiva na análise do comportamento da TSM ao longo de 40 anos nos seis pontos descritos. Para verificar a presença de tendências significativas nas séries foi utilizado o teste não-paramétrico de MK. $\mathrm{O}$ teste foi aplicado nas séries anuais de máximos, mínimos e médios da TSM, bem como nos valores sazonais para o nível de significância de $p$ valor $<0,05$.

Utilizou-se o estimador de declive de Sen para complementar as análises e o teste de Pettitt para verificar a não homogeneidade das séries. Os softwares Statistica e Statistica R foram utilizados nas análises.

(CV), assimetrias (A) e curtose (K) com os respectivos anos em que ocorreram os picos em cada ponto. Para os valores máximos anuais (Tabela 2) o ponto 6 apresenta o maior coeficiente de variação $(\mathrm{CV})$ seguido dos pontos 5 e 4 . Esses pontos estão localizados na plataforma continental próxima à costa da Argentina, região da $\mathrm{CBM}$. $\mathrm{O}$ ponto 6 apresenta valor de A próximo a zero, 
indicando uma distribuição mais próxima da normal. Nos demais pontos verificam-se tanto curvas assimétricas à direta (positivas) quanto à esquerda (negativas). Portanto, os valores de $\mathrm{K}_{\mathrm{s}}$ sugerem curvas platicúrticas (achatadas-valores negativos) em todos os pontos. Para os valores mínimos anuais (Tabela 3) verifica-se, também, maiores valores de $\mathrm{CV}$ nos pontos 4 e 5 , ou seja, maiores variações em torno da média, o que pode, também, ser explicado pela proximidade dos pontos na região da CBM, local com intensa instabilidade baroclínica e fortes gradientes latitudinais da TSM. Curvas assimétricas à esquerda são verificadas para todos os pontos exceto para o ponto 2, caracterizando não normalidade. No ponto 5 os valores indicam curva leptcúrtica (alongadapositiva) bem acentuada. Para os valores médios anuais (Tabela 4), o CV mostra que as séries apresentam pequenas oscilações em torno da média. As distribuições nos pontos $3,4,5$ e 6 têm curvas assimétricas à esquerda, enquanto no ponto 1 a distribuição é mais próxima da normal.

Tabela 2. Estatística descritiva dos valores máximos anuais da TSM $\left({ }^{\mathrm{a}} \mathrm{C}\right)$

\begin{tabular}{lccccccc}
\hline \multicolumn{1}{c}{ Ponto } & Máxima & Mínima & Média & dp & CV $(\%)$ & \multicolumn{1}{c}{ A } & K \\
\hline 1 (R. G. do Sul) & $28,1(2017)$ & $24,6(1979)$ & 26,6 & 0,8 & 3,1 & $-0,09$ & $-0,68$ \\
2 (R. G. do Sul) & $27,6(2014)$ & $23,6(1979)$ & 25,5 & 1,0 & 3,8 & 0,09 & $-0,76$ \\
3 (Uruguai) & $26,7(2010)$ & $23,0(1998)$ & 24,7 & 0,9 & 3,6 & $-0,07$ & $-0,48$ \\
4 (Foz do Prata) & $25,2(2004)$ & $21,1(1981)$ & 23,5 & 1,0 & 4,1 & $-0,04$ & $-0,30$ \\
5 (Argentina) & $21,7(2005)$ & $18,1(1988)$ & 19,8 & 0,9 & 4,5 & 0,16 & $-0,24$ \\
6 (Argentina) & $20,3(2004)$ & $16,9(2001)$ & 18,6 & 0,9 & 4,7 & 0,03 & $-0,47$ \\
\hline
\end{tabular}

Tabela 3. Estatística descritiva dos valores mínimos anuais da TSM $\left({ }^{\mathrm{a}} \mathrm{C}\right)$

\begin{tabular}{lccccccc}
\hline \multicolumn{1}{c}{ Ponto } & Máxima & Mínima & Média & dp & CV $(\%)$ & A & K \\
\hline 1 (R. G. do Sul) & $19,8(2015)$ & $15,6(1979)$ & 18,4 & 0,8 & 4,5 & $-0,10$ & $-0,44$ \\
2 (R. G. do Sul) & $18,0(2015)$ & $12,7(1979)$ & 16,0 & 1,0 & 6,1 & 1,16 & 1,58 \\
3 (Uruguai) & $15,8(2001)$ & $12,0(2007)$ & 13,8 & 1,0 & 7,1 & $-0,12$ & 0,09 \\
4 (Foz do Prata) & $13,5(2001)$ & $8,2(2016)$ & 10,9 & 1,2 & 10,9 & $-0,12$ & $-0,75$ \\
5 (Argentina) & $10,0(1998)$ & $6,8(1991)$ & 7,8 & 0,7 & 8,4 & $-0,88$ & 2,50 \\
6 (Argentina) & $9,3(1981)$ & $7,2(1984)$ & 8,3 & 0,6 & 6,5 & $-0,77$ & 2,16 \\
\hline
\end{tabular}

Tabela 4. Estatística descritiva dos valores médios anuais da TSM $\left({ }^{\mathrm{a}} \mathrm{C}\right)$

\begin{tabular}{lccccccc}
\hline \multicolumn{1}{c}{ Ponto } & Máxima & Mínima & Média & dp & CV $(\%)$ & A & K \\
\hline 1 (R. G. do Sul) & $23,1(2017)$ & $20,7(1979)$ & 22,4 & 0,5 & 2,4 & 0,04 & $-0,47$ \\
2 (R. G. do Sul) & $21,6(2017)$ & $18,6(1979)$ & 20,6 & 0,6 & 3,0 & 0,16 & 0,17 \\
3 (Uruguai) & $19,9(2017)$ & $17,5(1979)$ & 19,0 & 0,5 & 2,6 & $-0,69$ & 1,91 \\
4 (Foz do Prata) & $18,0(2002)$ & $14,9(2016)$ & 16,7 & 0,6 & 3,4 & $-1,08$ & 2,25 \\
5 (Argentina) & $14,1(1998)$ & $12,1(1988)$ & 13,1 & 0,4 & 3,3 & $-0,94$ & 1,88 \\
6 (Argentina) & $13,6(2004)$ & $12,1(1984)$ & 12,8 & 0,4 & 3,1 & $-1,06$ & 1,99 \\
\hline
\end{tabular}

O maior valor de K é verificado no ponto 4, mostrando uma distribuição leptcúrtica. Valores máximos anuais são verificados a partir do inicio dos anos 2000 em todos os pontos analisados, enquanto que os picos mínimos ocorreram a partir de 1979 até fins dos anos de 1990 exceto no ponto 6 (Tabela 2). Nos demais, verifica-se uma predominância de ocorrência de picos máximos, também, nos anos 2000 e mínimos a partir de 1979 e em alguns anos das décadas posteriores (Tabelas 3 e 4$)$.
Análises das Tendências da TSM de 1979 a 2018

Para as análises de tendências da TSM foram utilizados os testes de regressão linear, nãoparamétricos de MK, declividade de Sen e Pettitt. A Figura 3 mostra as anomalias da TSM máxima, mínima e média com as respectivas retas de tendência linear nos seis pontos. As retas de tendência mostram a ocorrência de mudança de patamar nas anomalias a partir do final da década de 1990 e inicio dos anos $2000 \mathrm{em}$ todos os pontos. No entanto, essas tendências não são conclusivas, pois podem estar associadas à dinâmica oceânica dessa região, na qual predominam as características 
relacionadas à CBM. Embora a partir do final da década de 1970 e início da década de 1980, a TSM começou a ser estimada com o auxílio de satélites ambientais, os dados mostram que as alterações nas anomalias vão se tornar mais pronunciadas a partir do século XXI. Observa-se ainda que, a TSM máxima mostra tendência positiva em todos os pontos. Já a TSM mínima não mostra tendência acentuada, exceto no ponto 4 onde a tendência
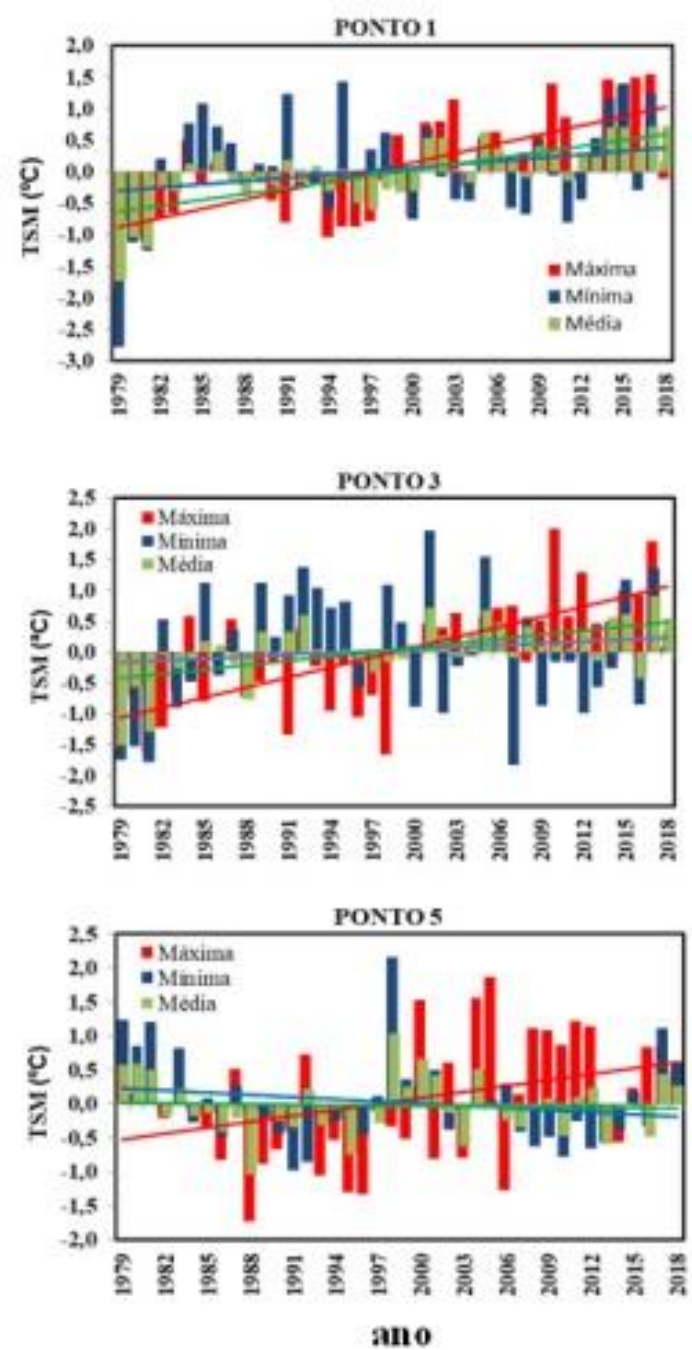

negativa se destaca das demais. Nas anomalias médias da TSM são somente observadas pequenas alterações positivas nos pontos $1,2,3$ e 6 . Ocorrências intensas de anomalias de TSM identificadas em regiões específicas que alteram padrões de circulação atmosférica, podem de certa forma estar associadas a alterações temporais e espaciais na corrente oceânica local. (da Silveira e Pezzi, 2014; Pezzi, 2016).
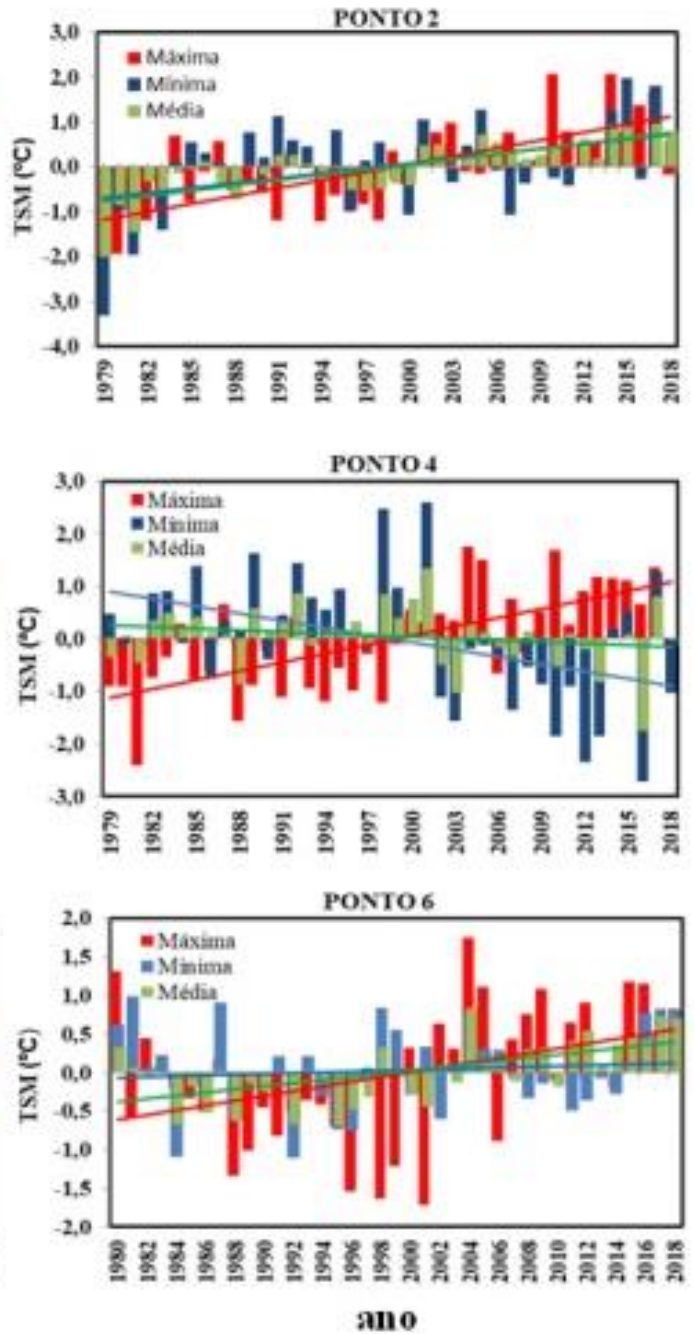

Figura 3. Anomalias da TSM máxima, mínima e média na região oceânica da plataforma continental referentes aos pontos entre o Rio Grande do Sul e a Argentina no período de 1979 a 2018, exceto no ponto 6 que teve início a partir de janeiro de 1980.

A região de estudo onde se encontra a CBM é caracterizada pela presença de massas d'água diferentes e as anomalias negativas de TSM podem estar associadas a vários fatores como o fluxo de água fria em direção ao norte, descarga do rio da Prata e processos de ressurgência. Já as anomalias positivas podem ter relação com a CB em direção mais ao sul como também as taxas de descarga do rio da Prata e interações oceano-atmosfera (da Silveira e Pezzi, 2014; Pezzi, 2016). Paniagua et al.,
(2018) mostram que grandes anomalias positivas na TSM são observadas durante o regime fraco da CM cujo impacto nos fluxos de calor entre atmosferaoceano podem afetar o clima local da região que possui grandes concentrações de clorofila/ano, e podem, portanto, afetar a distribuição da clorofila-a e sua produção primária associada. Um fator importante relacionado com anomalias positivas (negativas) da TSM é o aumento (redução) da precipitação no Sul do Brasil, interferindo também 
na atuação da ZCAS (Barros et al., 2000; Tomaziello e Gandu, 2013). Os primeiros autores mostram que anomalias positivas (negativas) de TSM entre $20^{\circ} \mathrm{S}$ e $40^{\circ} \mathrm{S}$ estão associadas com o deslocamento da ZCAS para sul (norte) que tende a ser mais fraca (intensa). Porém, quando atuam como forçante, as anomalias positivas tendem a intensificar a ZCAS e causar deslocamento para norte, enquanto que anomalias negativas tendem a enfraquecer (Barros et al., 2000; Chaves e Nobre, 2004).

Os gráficos da Figura 3 mostram ainda o padrão de tendência positiva da $\mathrm{TSM}_{\text {Máx }}$ a partir do final da década de 1990 e inicio dos anos 2000 com anomalias variando entre $1,5^{\circ} \mathrm{C}$ a $2,0^{\circ} \mathrm{C}$, aproximadamente, em todos os pontos. A continuidade dessas anomalias positivas podem ocasionar alterações nos processos meteoceanográficos, intensificando-os ou enfraquecendo-os e com isso provocar mudanças nos padrões climáticos da região. Segundo Freitas e Souza (2013), num cenário de aquecimento global na região da CBM, considerando período presente (1981-2000) e futuro (até 2100), anomalias positivas da TSM apresentaram tendência de aumento de $2,0{ }^{\circ} \mathrm{C}$ com maiores valores para cenários futuros. É importante ressaltar que no ano de 1979 são verificadas anomalias negativas acentuadas da TSM mínima e média nos pontos $1 \mathrm{e}$ 2 , em torno de $-3.0^{\circ} \mathrm{C}$, aproximadamente, sugerindo uma relação com processos meteoceanográficos conforme descritos anteriormente. Segundo Silva e Silva (2016) várias áreas de bacias oceânicas apresentam tendência positiva de acordo com análise espectral da TSM global de 1884 a 2014, caracterizando o Atlântico Sul como uma bacia que apresenta sinais de aumento da energia espectral na faixa de oscilação, de 1 a 12 meses. Outra característica importante encontrada ao longo do período é que fenômenos caracterizados por oscilações mais lentas (entre 4 a 8 e 8 a 12 anos) têm se tornado mais energéticos.
Na Tabela 5 são apresentados os resultados dos testes de tendência linear e não-paramétricos aplicados às séries máximas, mínimas e médias anuais da TSM. Observa-se que os resultados tanto para os escores TAU (MK) como para os coeficientes angulares apresentam os mesmos sinais referentes às tendências crescentes ou decrescentes, ou seja, positivas ou negativas, respectivamente. Para as $\mathrm{TSM}_{\text {Máx, os testes }}$ mostram valores maiores que zero, sugerindo tendência crescente para todos os pontos, exceto para o ponto 5 que não apresentou tendência significativa. Os maiores valores de TAU foram observados nos pontos 4, 3, 2 e 1, com valores de declividade Sen de aproximadamente $0,06{ }^{\circ} \mathrm{C} / \mathrm{ano}$, o que representa tendências crescentes com valores entre $2{ }^{\circ} \mathrm{C}$ a $2,5{ }^{\circ} \mathrm{C} / 40$ anos. Para os valores de TSM $_{\text {Mín, os escores TAU apresentam valores baixos }}$ e $p$ valor maiores do que 0,05 , indicando tendência não significativa, exceto para o ponto 4 que apresenta valor para Sen de $-0,05 /$ ano e tendência decrescente de $-2{ }^{\circ} \mathrm{C} / 40$ anos. Os resultados dos testes para a $\mathrm{TSM}_{\text {Méd }}$ mostram tendências positivas significativas para os pontos $6,3,2$ e 1 com valores de Sen em torno de 0,03/ano e tendências crescentes de aproximadamente $1^{\circ} \mathrm{C} / 40$ anos. Para o ponto 4 os testes apresentam significância mas o valor de Sen está próximo a zero $(-0,006)$ com tendência decrescente em torno de $-0,3{ }^{\circ} \mathrm{C} / 40$ anos.

Na Figura 4 são apresentados os gráficos do teste MK aplicado aos dados de TSM máxima, mínima e média para os respectivos pontos, no qual pode ser observada uma semelhança com os gráficos de anomalia da Figura $3 . \quad$ Também

apresentam os pontos de mudança nas séries máximas e médias indicadas pela linha preta vertical com significância $p>0,05$. Para a série de mínimos, somente no ponto 4 foi identificada alteração na média com transição descendente. 
Tabela 5. Testes estatísticos aplicados às séries temporais anuais da TSM para os seis pontos no período de 1979 a 2018 (ponto 6 de 1980 a 2018).

\begin{tabular}{|c|c|c|c|c|c|c|}
\hline Mínima & Ponto 6 & Ponto 5 & Ponto 4 & Ponto 3 & Ponto 2 & Ponto 1 \\
\hline Reg. Linear (coef. ang) & 0,05 & $-0,01$ & $-0,05$ & 0,01 & 0,02 & 0,02 \\
\hline TAU & 0,0361 & $-0,162$ & $-0,32$ & 0,0571 & 0,213 & 0,0677 \\
\hline$p$ valor $<0.05$ & 0,7613 & 0,1536 & 0,0042 & 0,6158 & 0,0583 & 0,5516 \\
\hline $\operatorname{Sen}\left({ }^{\circ} \mathrm{C} / \mathrm{ano}\right)$ & 0 & $-0,0111$ & $-0,05$ & 0,0083 & 0,0288 & 0,0080 \\
\hline Tend $_{\text {TSM }}\left({ }^{\circ} \mathrm{C} / 40\right.$ anos $)$ & 0 & $-0,444$ & $-2,0$ & 0,332 & 1,152 & 0,32 \\
\hline Máxima & Ponto 6 & Ponto 5 & Ponto 4 & Ponto 3 & Ponto 2 & Ponto 1 \\
\hline Reg. Linear (coef. ang) & 0,03 & 0,03 & 0,05 & 0,05 & 0,06 & 0,05 \\
\hline TAU & 0,275 & 0,192 & 0,481 & 0,55 & 0,514 & 0,494 \\
\hline$p$ valor $<0.05$ & 0,0149 & 0,0862 & $1,73 \mathrm{E}-05$ & $1,07 \mathrm{E}-06$ & 4,29E-06 & $1,01 \mathrm{E}-05$ \\
\hline $\operatorname{Sen}\left({ }^{\circ} \mathrm{C} / \mathrm{ano}\right)$ & 0,0312 & 0,0241 & 0,0574 & 0,0538 & 0,0620 & 0,05 \\
\hline Tend $_{\text {TSM }}\left({ }^{\circ} \mathrm{C} / 40\right.$ anos $\left.)\right)$ & 1,217 & 0,964 & 2,296 & 2,152 & 2,48 & 2,0 \\
\hline Média & Ponto 6 & Ponto 5 & Ponto 4 & Ponto 3 & Ponto 2 & Ponto 1 \\
\hline Reg. Linear (coef. ang) & 0,02 & $-0,002$ & $-0,01$ & 0,02 & 0,04 & 0,03 \\
\hline TAU & 0,425 & $-0,0317$ & $-0,168$ & 0,393 & 0,553 & 0,503 \\
\hline$p$ valor $<0.05$ & 0,0002 & 0,7877 & $1,41 \mathrm{E}-01$ & $5,26 \mathrm{E}-04$ & $8,34 \mathrm{E}-07$ & $8,70 \mathrm{E}-06$ \\
\hline $\operatorname{Sen}\left({ }^{\circ} \mathrm{C} / \mathrm{ano}\right)$ & 0,02 & 0 & $-0,006$ & 0,0215 & 0,0348 & 0,0272 \\
\hline $\operatorname{Tend}_{\mathrm{TSM}}\left({ }^{\circ} \mathrm{C} / 40\right.$ anos $\left.)\right)$ & 0,78 & 0 & $-0,276$ & 0,86 & 1,392 & 1,088 \\
\hline
\end{tabular}

A Tabela 6 apresenta os resultados do teste de Petttit no qual a hipótese nula utilizada foi de que não há mudança na média dos dados e a hipótese alternativa a de que existe certo dado no qual um ponto de mudança pode ser detectado e a média do conjunto de dados muda nesse ponto de interrupção. Pontos de mudança estatisticamente significativos foram detectados para a TSM Máx nos pontos de grade 1 e 2 no ano 2000, nos pontos 3 e 4 em 1998, no ponto 5 em 1999 e no ponto 6 em 2001 com transição da média ascendente. Com relação à $\mathrm{TSM}_{\text {Méd, }}$, todos os pontos de grade apresentaram ponto de mudança, exceto o ponto 5 , os pontos 1,2 e 3 no ano 2000, ponto 4 em 2001 e 6 em 2003 com transição ascendente, exceto para o ponto 3 . Para a TSM $_{\text {Mín, }}$ o estimador somente identificou ponto de mudança ascendente no ponto 4, em 2001. 

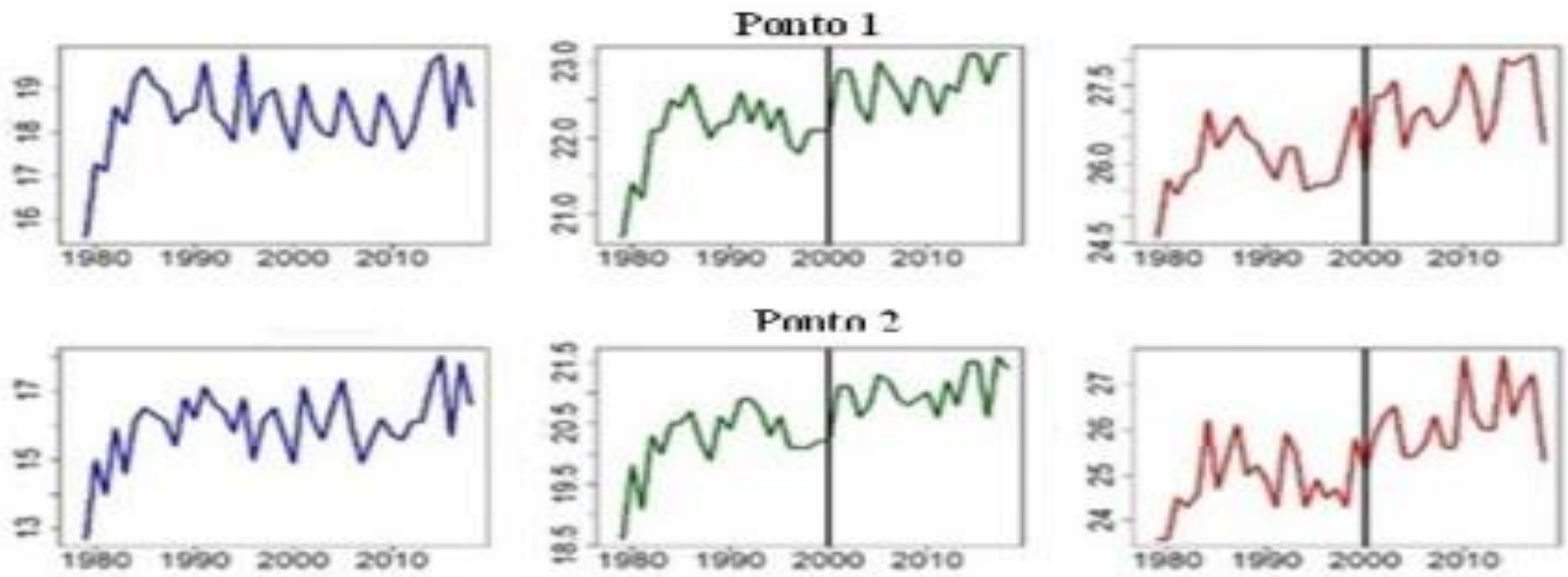

Ponto 2
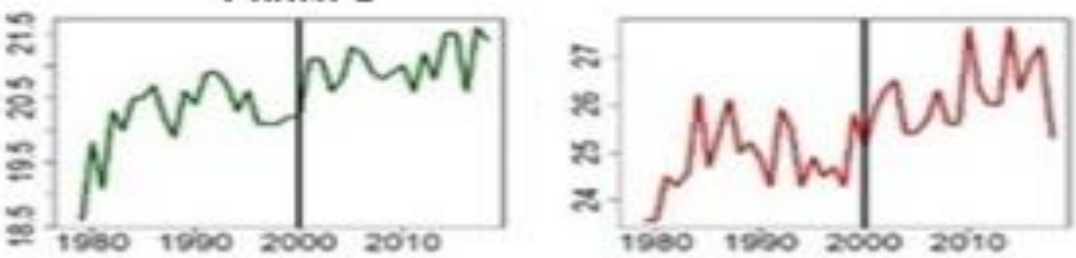

Ponto 3
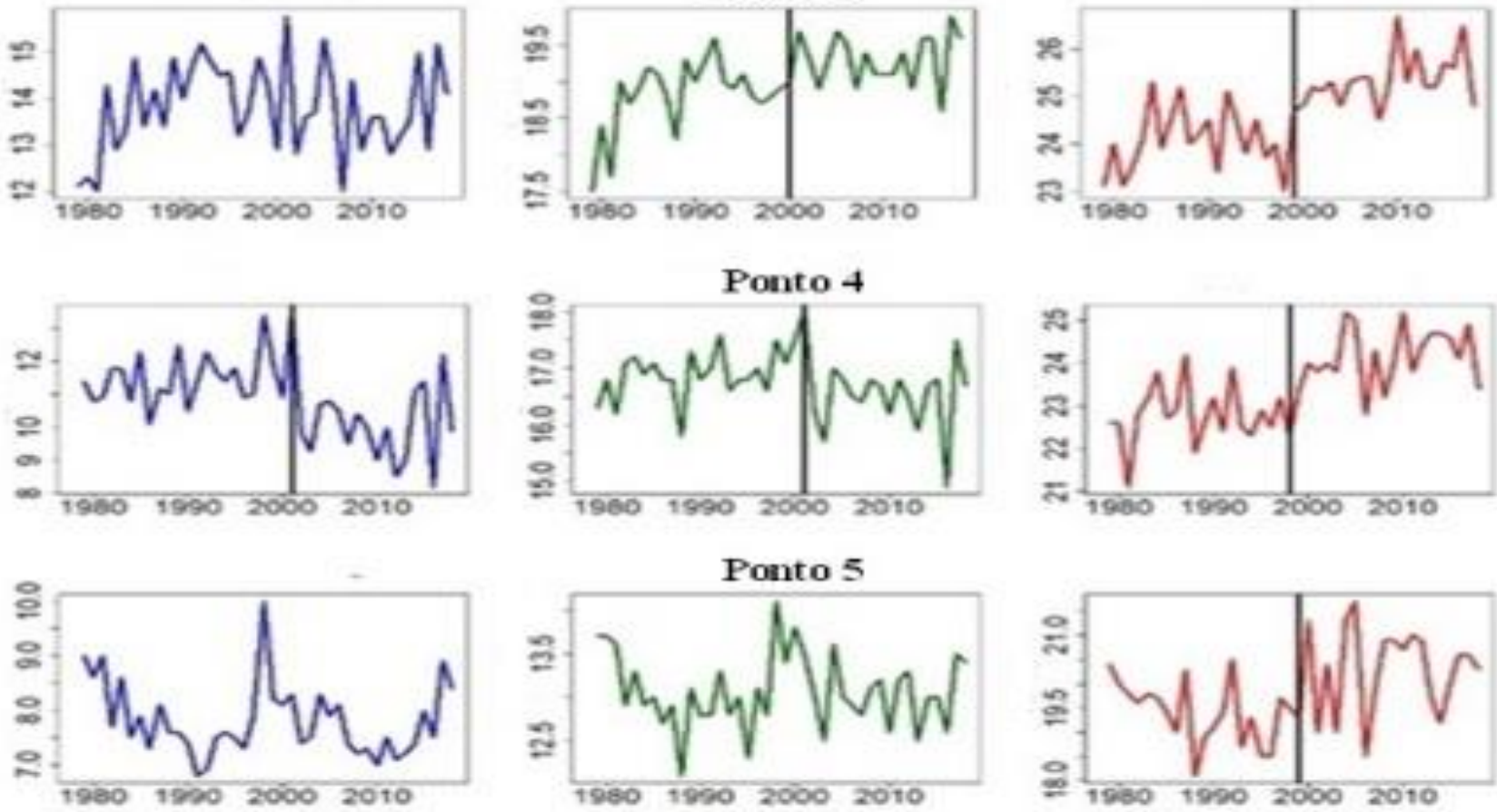

Ponto 5
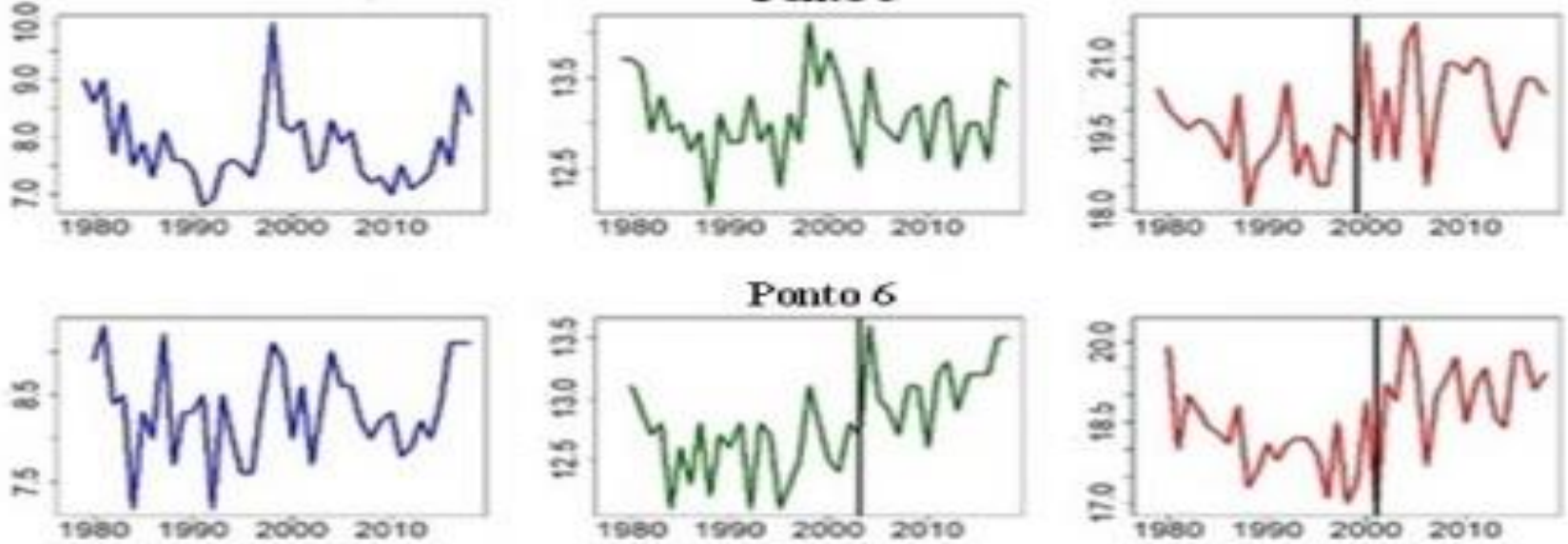

Figura 4: Séries temporais das TSM máximas (vermelho), mínimas (azul) e médias (verde) a partir dos testes de MK e o ponto de mudança (linha preta) com o Statistica $\mathrm{R}$ para os seis pontos.

Oliveira, M. M. F., Oliveira, J. L. F., Fernandes, P. J. F., Ebecken, N. F. F. 
Tabela 6. Resultados do teste de Pettitt com os $p$-valores significativos em negrito, o ano do ponto de mudança e a transição da média.

\begin{tabular}{cccc}
\hline Ponto & $p$-valor & Ano de mudança & Transição \\
\hline 1-Mín & 0,622 & $\mathrm{NS}$ & \\
1-Méd & $7,40 \times 10^{-5}$ & 2000 & ascendente \\
1-Máx & $9,44 \times 10^{-5}$ & 2000 & ascendente \\
\hline 2-Mín & 0,277 & $\mathrm{NS}$ & \\
2-Méd & $2,72 \times 10^{-5}$ & 2000 & ascendente \\
2-Máx & 0,0001 & 2000 & ascendente \\
\hline 3-Mín & 0,396 & $\mathrm{NS}$ & \\
3-Méd & 0,008 & 2000 & ascendente \\
3-Máx & $2,90 \times 10^{-5}$ & 1998 & ascendente \\
\hline 4-Mín & 0,005 & 2001 & ascendente \\
4-Méd & 0,042 & 2001 & descendente \\
4-Máx & $5,44 \times 10^{-5}$ & 1998 & ascendente \\
\hline 5-Mín & 0,350 & $\mathrm{NS}$ & \\
5-Méd & 0,757 & $\mathrm{NS}$ & ascendente \\
5-Máx & 0,013 & 1999 & \\
6-Mín & 0,775 & $\mathrm{NS}$ & ascendente \\
6-Méd & 0,0004 & 2003 & ascendente \\
6-Máx & 0,0008 & 2001 & \\
\hline
\end{tabular}

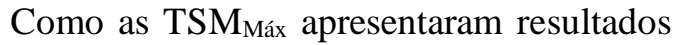
de tendência positivas para todos os pontos foi, então, realizada uma análise de correlação (Tabela 7) entre as séries dos respectivos pontos para avaliar o grau de dependência entre as variáveis na região de domínio da CBM, uma vez que os sistemas meteoceanográficos desenvolvidos nessa região influenciam as Regiões Sul e Sudeste do Brasil. Segundo (Leyba et al., 2017) existe uma dependência regional de fluxos de calor associados a vórtices de mesoescala na região da CBM onde uma ligação significativa entre as anomalias da TSM geradas por esses vórtices e o fluxo anômalo de calor local afetam as camadas mais baixas da atmosfera.

Os coeficientes de correlação com nível de significância $p<0,05$ apresentam valores entre 0,84 a 0,64 para os pontos 1,2 e 3 correlacionados com o ponto 4 (CBM). Os pontos 5 e 6 também apresentam valores significativos de correlação em relação ao ponto 4 de 0,62 e 0,56 , respectivamente, porém apresentam maior valor de correlação entre eles, sugerindo a influência da região sob o domínio da CM. O coeficientes de correlação máximo de
0,89 foi obtido entre os pontos 2 e 1 , seguido dos pontos 3 e 2 com valor de 0,87 e 4 e 3 com valor de 0,84 , o que pode ser interpretado como uma resposta ao fato de que a região onde se localizam esses pontos encontra-se sob o regime de influência da CB. O ponto 4 , contudo, mostra correlação com os demais pontos, porém os maiores valores são observados tanto nos pontos localizados na região da CB quanto na CM. Dessa forma, portanto, através da matriz de correlação entre os respectivos pontos referentes aos dados da TSM $_{\text {Máx }}$ é possível identificar a presença de sistemas distintos na região, sendo que no ponto 4 a correlação significativa com os demais, aponta para uma dependência de ambos os sistemas marinhos.

Com base nos resultados obtidos com o teste MK para os valores máximos anuais da TSM (Tabela 5) foi verificado, também, o Noc de extremos acima do percentil de 90 das séries completas ( 6 em 6 horas) no período de estudo para os pontos $6,4,3,2$ e 1 (Tabela 8 ), os quais apresentaram nível de significância $p<0,05)$ A Tabela 9 mostra os valores de ocorrências sazonais. 
Tabela 7. Matriz de correlação das TSM máximas entre os seis pontos com nível de significância de $\mathrm{p}<0,005$.

\begin{tabular}{cccccc}
\hline Pontos & P6 & P5 & P4 & P3 & P2 \\
\hline 5 (Argentina) & 0,76 & & & & \\
4 (Foz do Prata) & 0,56 & 0,62 & & & \\
3 (Uruguai) & 0,42 & 0,43 & 0,84 & & \\
2 (R. G. do Sul) & 0,23 & 0,28 & 0,74 & 0,87 & \\
1 (R. G. do Sul) & 0,23 & 0,22 & 0,64 & 0,78 & 0,89 \\
\hline
\end{tabular}

Tabela 8. Noc decadal de valores da TSM acima do percentil de 90 para os pontos 6, 4, 3, 2 e 1.

\begin{tabular}{cccccc}
\hline Período & $\mathrm{P} 6\left(18,4^{\circ} \mathrm{C}\right)$ & $\mathrm{P} 4\left(22,0^{\circ} \mathrm{C}\right)$ & $\mathrm{P} 3\left(23,5^{\circ} \mathrm{C}\right)$ & $\mathrm{P} 2\left(24,1^{\circ} \mathrm{C}\right)$ & $\mathrm{P} 1\left(25,2^{\circ} \mathrm{C}\right)$ \\
\hline $79-80$ & 128 & 312 & 88 & 156 & 156 \\
$81-90$ & 68 & 1617 & 1176 & 1726 & 2548 \\
$91-00$ & 56 & 1117 & 844 & 1344 & 1692 \\
$01-10$ & 631 & 1614 & 1852 & 2987 & 3255 \\
$11-18$ & 719 & 1485 & 1908 & 2459 & 2822 \\
\hline $79-18$ & 1602 & 6145 & 5868 & 8672 & 10473 \\
\hline
\end{tabular}

Tabela 9. Noc sazonal de valores da TSM acima do percentil de 90 para os pontos 6, 4, 3, 2 e 1 com os valores superiores das séries da TSM máximas de $6 \mathrm{em} 6$ horas.

\begin{tabular}{cccccc}
\hline Mês & $\mathrm{P} 6\left(18,4^{\circ} \mathrm{C}\right)$ & $\mathrm{P} 4\left(22,0^{\circ} \mathrm{C}\right)$ & $\mathrm{P} 3\left(23,5^{\circ} \mathrm{C}\right)$ & $\mathrm{P} 2\left(24,1^{\circ} \mathrm{C}\right)$ & $\mathrm{P} 1\left(25,2^{\circ} \mathrm{C}\right)$ \\
\hline Dezembro & 0 & 103 & 54 & 80 & 151 \\
Janeiro & 399 & 1954 & 1479 & 2092 & 2628 \\
Fevereiro & 795 & 2590 & 2483 & 2998 & 3326 \\
Março & 408 & 1451 & 1792 & 3147 & 3568 \\
Abril & 0 & 47 & 60 & 344 & 796 \\
Maio & 0 & 0 & 0 & 1 & 4 \\
\hline Total & 1602 & 6145 & 5868 & 8672 & 10473 \\
\hline
\end{tabular}

Na Tabela 8 observa-se que entre 2001 e 2018 os valores da TSM $_{\text {Máx }}$ ultrapassaram o percentil de 90 em todos os pontos da região de estudo. Porém, os maiores (menores) valores ocorreram no ponto 1 (ponto 6), ou seja, nas coordenadas de -29,37/-48,05, Rio Grande do Sul (coordenadas de -42,80/-60,38, Argentina).

No ponto 6 ocorreu um aumento significativo no período em comparação com as décadas anteriores. Essa análise permite inferir que a partir da década de 90 ocorreu um aumento no Noc de extremos em todos os pontos analisados.

A Tabela 9 apresenta o Noc sazonal, na qual se verifica que os maiores valores acima do percentil de 90 ocorreram nos meses de verão (janeiro, fevereiro e março) em todos os pontos, sendo que o mês de fevereiro concentra o maior número de ocorrências nos pontos 6,4 e 3 . Nos pontos 2 e 1 as ocorrências no mês de março superam os valores ocorridos em fevereiro. $\mathrm{O}$ mês de abril (outono) mostra um aumento gradativo a partir do ponto 4 , chegando a alcançar mais de $100 \%$ no ponto 1 em relação ao ponto 2 , ambos no RGS. Também, se verifica nesses dois pontos ocorrências no mês de maio (outono) de 2018.

A Figura 5 apresenta as flutuações anuais do Noc acima do percentil de 90. As médias de ocorrências para o período de 40 anos nos pontos 6 , 4, 3, 2 e 1 são de, aproximadamente, 41, 154, 147, 217 e 262, respectivamente. Tendências lineares positivas são identificadas em todos os pontos. Observa-se, também, que no ponto 6 os valores mais extremos começam a ocorrer entre 1998 e 2000. Nos pontos 3, 2 e 1, as flutuações são mais acentuadas, ocorrendo maiores valores, também, a partir do ano 2000. A equação de regressão linear mostra que os coeficientes angulares das retas (a) são maiores para o ponto 2 , seguido do ponto 1 e 3 , indicando maiores tendências para os respectivos pontos. 

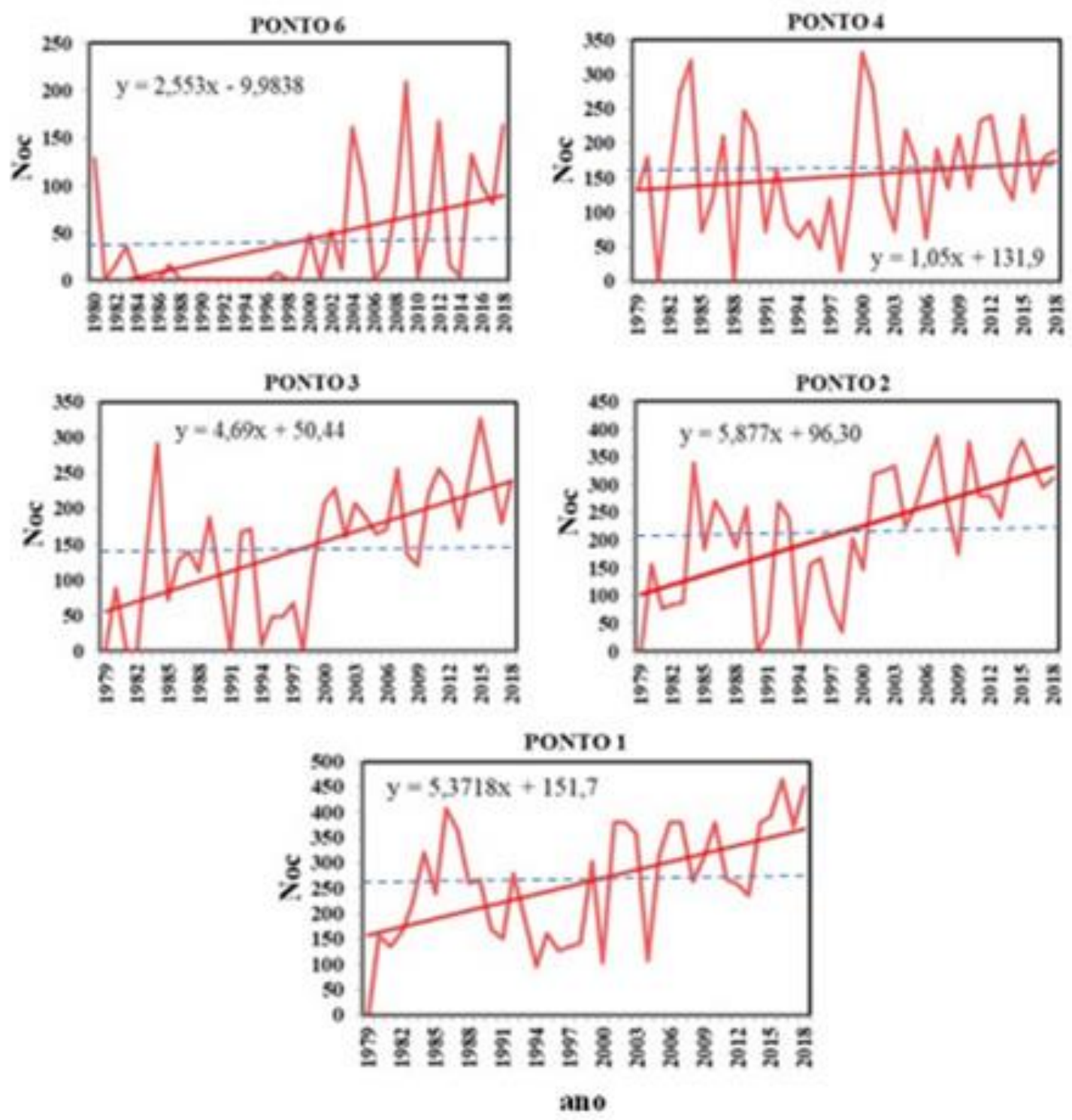

Figura 5. Séries temporais das flutuações anuais do Noc da TSM Máx acima do percentil de 90 nos pontos 6, 4, 3, 2 e 1 no período de 40 anos. As linhas azuis tracejadas indicam as médias de ocorrência no período e as linhas retas em vermelho mostram as tendências positivas em cada ponto.

Continuando a verificação do comportamento da TSM na região de estudo devido às tendências positivas encontradas nos valores máximos, optou-se em verificar, também, as tendências sazonais. Conforme mostra a Tabela 9, os maiores valores do Noc sazonal referentes aos máximos da TSM acima do percentil de 90 para os pontos $6,4,3$, 2 e 1 foram verificados nos meses de janeiro, fevereiro e março (verão). Portanto, os testes MK e Sen foram aplicados, também, para esse período, considerando-se a média dos três meses durante os 40 anos. Os resultados são apresentados na Tabela 10 onde se verifica significância para os pontos 3,2 e 1 com tendências positivas, aproximadamente, entre $1{ }^{\circ} \mathrm{C}$ a $2{ }^{\circ} \mathrm{C}$. 
Tabela 10: Testes não-paramétricos de MK e estimador de Sen aplicados aos valores médios da TSMMáx referentes aos meses de janeiro, fevereiro e março (40 anos).

\begin{tabular}{c|c|c|c|c|c|c}
\hline TSM_MÁX (JFM) & P 6 & P 5 & P 4 & P 3 & P 2 & P 1 \\
\hline TAU & 0.2064 & 0.0474 & 0.1820 & 0.5230 & 0.4051 & 0.3717 \\
p valor <0.05 & 0.0652 & 0.6739 & 0.0994 & $2.019 \mathrm{e}-06$ & 0.00023 & 0.00073 \\
Sen $\left({ }^{\circ} \mathrm{C} /\right.$ ano $)$ & 0.0190 & 0.0028 & 0.0133 & 0.04285714 & 0.04 & 0.0285 \\
TSM $\left({ }^{\circ} \mathrm{C} / 40\right.$ anos) & 0.74 & 0.11 & 0.54 & 1.71 & 1.6 & 1.14 \\
\hline
\end{tabular}

\section{Conclusões}

$\mathrm{O}$ oceano e a atmosfera interagem acoplados, trocando matéria e energia, de forma, que não podem ser considerados separadamente. $\mathrm{O}$ oceano varia lentamente, enquanto a atmosfera varia rapidamente. $\mathrm{O}$ fluxo de vapor na interface oceano-atmosfera está intimamente relacionado à TSM cujo gradiente varia ao longo da latitude, relacionado a processos oceânicos e atmosféricos como fonte de energia potencial na atmosfera, contribuindo com a formação e desenvolvimento de todas as classes de ciclogênese.

Aumentos na TSM reduzem o oxigênio dissolvido o que afeta significativamente a vida marinha, alterando de forma prejudicial à cadeia trófica de organismos sensíveis à temperatura e às propriedades químicas inerentes aos ambientes marinhos como já vem sendo identificado na costa sul e sudeste do país com a sardinha verdadeira (Sardinella brasiliensis).

Na região da CBM até o Rio Grande do Sul está ocorrendo uma tendência positiva no padrão de máximos da TSM. Entretanto, essa tendência não é conclusiva e pode estar associada tanto a variabilidade na dinâmica oceânica quanto antrópica, porém o contínuo aumento de máximos da TSM pode levar ao desenvolvimento de sistemas meteorológicos extremos na região, o que já vem sendo observado nos últimos anos, também, nas Regiões Sul e Sudeste do Brasil. Por sua vez, mínimos da TSM não mostram tendências acentuadas, exceto na Foz do Prata (ponto 4) na região da $\mathrm{CBM}$, onde a tendência negativa se destaca dos demais.

A série de TSM anual, por sua vez, não é estacionária nos seis pontos analisados, pois apresenta tendência de crescimento. Essas tendências iniciaram, aproximadamente, no final da década de 1990 e início dos anos 2000, o que está de acordo com pesquisas recentes que mostram um padrão de aquecimento superficial a partir do início do século XXI, evoluindo nos últimos anos com o maior aquecimento já registrado. As tendências positivas encontradas nas anomalias e nos Noc de extremos anuais (acima percentil de 90), também, vêm ocorrendo desde o ano 2000.

\section{Agradecimentos}

À Fundação de Amparo à Pesquisa do Estado do Rio de Janeiro (FAPERJ) pelo apoio financeiro e a contribuição dos revisores anônimos no sentido de aprimorar a qualidade do manuscrito.

\section{Referências}

Abram, N., J.-P. Gattuso, A. Prakash, L. Cheng, M.P. Chidichimo, S. Crate, H. Enomoto, M. Garschagen, N. Gruber, S. Harper, E. Holland, R.M. Kudela, J. Rice, K. Steffen, and K. von Schuckmann, 2019: Framing and Context of the Report. In: IPCC Special Report on the Ocean and Cryosphere in a Changing Climate.

Pörtner, D.C. Roberts, V. Masson-Delmotte, P. Zhai, M. Tignor, E. Poloczanska, K. Mintenbeck, A. Alegría, M. Nicolai, A. Okem, J. Petzold, B. Rama, N.M. Weyer (eds.)]. In press.

Alexandre, G.R., Baptista, M.B., Naghettini, M.C., 2010. Estudo para identificação de tendências do regime pluvial na Região Metropolitana de Belo Horizonte a partir de métodos estatísticos. Revista Brasileira de Recursos Hídricos 15, 115 136.

Arikan, B.B., Kahya, E., 2019. Homogeneity revisited: analysis of updated precipitation series in Turkey. Theoretical Applied Climatology 135, 211-220. Doi: 10.1007/s00704-018-2368$\mathrm{x}$.

Aristegui, J., Guinder, V.A., Halberg, R., Hilmi, N., Jiao, N., Karim, M.S., Levin, L., O’Donoghue, T.S., Cuicapusa, S.R.P., Rinkevich, B., Suga, T., Tagliabue; A., Willamson, P., 2019. Changing Ocean Marine Ecosystems, and Dependent Communities, in: Bindoff, N.L., Cheung, W.W.L., Kairo, J.G., IPCC SR Ocean and Cryosphere; Axel Durand, Australia, pp. 5-198.

Back, A.J., 2001. Aplicação de análise estatística para identificação de tendências climáticas. Pesquisa Agropecuária Brasileira, Brasília 36, 717-726. 
BANCO MUNDIAL, 2012a. Avaliação de Perdas e Danos: Inundações Bruscas em Santa CatarinaNovembro de 2008. Brasília: BM.

BANCO MUNDIAL, 2012b. Avaliação de Perdas e Danos: Inundações e Deslizamentos na Região Serrana do Rio de Janeiro - Janeiro de 2011. Brasília: BM.

Barros, V., Gonzalez, M., Liebmann, B., 2000. Influence of the South Atlantic Convergence Zone and South Atlantic sea surface temperature on interannual summer rainfall variability in Southeastern South America. Theoretical and Applied Climatology 67, 123-133.

Berrisford, P., Dee, D.P., Poli, P., Brugge, R., Fielding, M., Fuentes, M., Kållberg, P.W., Kobayashi, S., Uppala, S., 2011. The ERAInterim archive Version 2.0, ERA Report Series, Shinfield Park, Reading, United Kindon, http://www.ecmwf.int/node/8174.

Berz, W., Kron, G., Loster, T., Rauch, E., Schimetschek, J., Schmieder, J., Siebert, A., Smolka, A., Wirtz, A., 2001. World map of natural hazards - a global view of the distribution and intensity of significant exposures. Natural Hazards 23, 443-465.

Blain, G.C., 2011. Considerações estatísticas relativas a seis séries mensais de temperatura do ar da Secretaria de Agricultura e Abastecimento do Estado de São Paulo. Revista Brasileira de Meteorologia 26, 279-296.

Bombardi, R.J., Carvalho, L.M.V. de, 2017. Práticas Simples em Análises Climatológicas: Uma Revisão. Revista Brasileira de Meteorologia 32, 311-320. Doi: http://dx.doi.org/10.1590/0102-77863230001.

Chaves, R.R., Nobre, P., 2004. Interactions between sea surface temperature over the South Atlantic Ocean and the South Atlantic Convergence Zone. Geophysical Research Letters 31, L03204.

Doi: http://doi.org/10.1029/2003GL018647.

Cheng, L., Zhu, J., Abraham, J., Trenberth, K.E., Fasulo, J.T., Zhang, B., Yu, F., Wan, L., Chen, X., Song, X., 2019. 2018 Continues Record Global Ocean Warming. Advances in Atmospheric Sciences 36, 249-252. Doi: http://doi.org/10.1007/s00376-019-8276-x.

Cheng, L. et al., 2020. Record-Setting Ocean Warmth Continued in 2019. Advances in Atmospheric Sciences 37, 137-142. http://doi.org/10.1007/s00376-020-9283-7.

Collins, M., Minobe, S., Barreiro, M., Bordoni, S., Kaspi, Y., Kuwano-Yoshida, A., et al., 2018. Challenges and opportunities for improved understanding of regional climate dynamics. Nature Climate Change 8(2), 101-108. Doi: http://doi.org/10.1038/s41558-017-0059-8.

D’Agostino, R., Lionello, P., 2017. Evidence of global warming impact on the evolution of the Hadley Circulation in ECMWF centennial reanalyzes, Climate Dynamics 48, 3047-3060. Doi: 10.1007/s00382-016-3250-0.

da Silva, R.M, Santos, C.A.G., Moreira, M, CorteReal, J., Silva, V.C., Medeiros, I.C., 2015. Rainfall and river flow trends using MannKendall and Sen's slope estimator statistical tests in the Cobres River basin. Natural Hazards 77, 1205-1221. Doi: http://doi.org/10.1007/s11069-015-1644-7.

da Silveira, I.P., Pezzi, L.P., 2014. Sea surface temperature anomalies driven by oceanic local forcing in the Brazil-Malvinas Confluence. Ocean Dynamics 64, 347-360. Doi: 10.1007/s10236-014-0699-4.

Dee, D.P., Uppala, S.M., Simmons, A.J., Berrisford, P., Poli, P., 2011. The ERA-Interim reanalysis: configuration and performance of the data assimilation system. Bulletin of American Meteorological Society 137, 553-597. https://doi.org/10.1002/qj.828.

Diaz, A.F., Studzinski, C.A., Mechoso, C.R., 1998. Relationships between precipitation anomalies in Uruguay and Southern Brazil and sea surface temperature in the Pacific and Atlantic Oceans. Journal of Climate 11, 251-271. Doi: http://doi.org/10.1175/15200442(1998)011<0251:RBPAIU>2.0.CO;2.

Di Giulio, G. M., Torres, R. R., Vasconcellos, M. P., Braga, D. R. G. C., Mancini, R. M., Lemos, M. C., 2019. Eventos extremos, mudanças climáticas e adaptação no Estado de São Paulo. Ambiente \& Sociedade 22, 1-20. Doi: http://doi.org/10.1590/18094422asoc0277r1vu1914ao.

Doyle, M. E., Barros, V.R., 2002. Midsummer lowlevel circulation and precipitation in subtropical South America and related sea surface temperature anomalies in the South Atlantic. Journal of Climate 15, 3394-3410. Doi: http://doi.org/10.1175/15200442(2002)015<3394:MLLCAP>2.0.CO;2.

Easterling, W.E., Aggarwal, P.K, Batima, P. Brander, K.M., Erda, L., Howden, S.M., Kirilenko, A., Morton, J., Soussana, J. F., Schmidhuber, J., Tubiello, F.N., 2007a. Food, fibre and forest products, in: Parry, M.L., Canziani, O.F., Palutikof, J.P., van der Linden, P.J., Hanson, C.E. (Eds.), Climate Change: 
Impacts, Adaptation and Vulnerability. Contribution of Working Group II to the Fourth Assessment Report of the Intergovernmental Panel on Climate Change. Cambridge University Press, Cambridge, pp. 273-313.

Easterling, W.E., 2007b. Climate change and the adequacy of food and timber in the 21 st century. PNAS, 104, 19679.

Freitas, R.A.P. de, Souza, R.B. de, 2013. Influência das anomalias da temperatura da superfície do mar em regiões de latitudes médias e altas na trajetória preferencial dos ciclones extratropicais sob condições climáticas de aquecimento global. Revista Ciência e Natura, Edição Especial, 524528.

Freitas, R.A.P. de, Souza, R.B. de, Reis, R.A.N. do, Lindemann, D.S. da, 2019. Relação entre o Vapor D’Água Atmosférico e a Temperatura da Superfície do Mar Sobre a Região da Confluência Brasil-Malvinas com Base em Dados Coletados In Situ. Revista Brasileira de Geografia Física 12, 1687-1702. Doi: 10.26848/rbgf.v12.5.p1687-1702.

Gavrilov, M.B, Tosic, I., Markovic, S.B., Unkasevic, M., Petrovic, P., 2016. Analysis of annual and seasonal temperature trends using the Mann-Kendall test in Vojvodina, Serbia. Időjárás-Quarterly. Journal of the Hungarian Meteorological Service 120, 183-198.

Gigliotti, E.S., Gherardi, D.F.M., Paes, E.T., Souza, R.B., Katsuragawa, M., 2010. Spatial analysis of egg distribution and geographic changes in the spawning habitat of the Brazilian sardine Sardinella brasiliensis. Journal of Fish Biology 77, 2248-2267. Doi: http://doi.org/10.1111/j.10958649.2010.02802.x.

Haylock, M.R., Peterson, T.C., Alves, L.M., Ambrizzi, T., Anunciação, Y.M.T., Baez, J., Barros, V.R., Berlato, M.A., Bidegain, M., Coronel, G., CorradI, V., Garcia, V.J., Grimm, A.M., Karoly, D., Marengo, J.A., Marino, M.B., Moncunill, D.F., Nechet, D., Quintana, J., Rebello, E., Rusticucci, M., Santos, J. L., Trebejo, I., Vincent, L.A., 2006. Trends in Total and Extreme South American Rainfall in 19602000 and Links with Sea Surface Temperature. Journal of Climate 19, 1490-1512. Doi: http://doi.org/10.1175/JCLI3695.1.

Hoskins, B.J., Hodjes, K.I., 2005. A New Perspective on Southern Hemisphere Storm Tracks. Journal of Climate 18, 4108-4129. Doi: http://doi.org/10.1175/JCLI3570.1.
IPCC, 2019: Summary for Policymakers. In: IPCC Special Report on the Ocean and Cryosphere in a Changing Climate [H.-O. Pörtner, D.C. Roberts, V. Masson-Delmotte, P. Zhai, M. Tignor, E. Poloczanska, K. Mintenbeck, A. Alegría, M. Nicolai, A. Okem, J. Petzold, B. Rama, N.M. Weyer (eds.)]. In press.

Irannezhad, M., Marttila, H., De-liang, C., Kløve, B., 2016. Century-long variability and trends in daily precipitation characteristics at three Finnish stations. Advances in Climate Change Research 7, 54-69 Doi: http://doi.org/10.1016/j.accre.2016.04.004.

Kendall, M G., 1975. Rank correlation measures. Charles Griffin: London, U.K, p. 220

Killick, R., Fearnhead, P., Eckley, I.A., 2012. Optimal Detection of Changepoints With a Linear Computational Cost. Journal of the American Statistical Association 107, 15901598. Doi: 10.1080/01621459.2012.737745.

Kocsis, T., Székely, I.K., Anda, A., 2020. Homogeneity tests and non-parametric analyses of tendencies in precipitation time series in Keszthely, Western Hungary. Theoretical and Applied Climatology139, 849-859. Doi: http://doi.org/10.1007/s00704-019-03014-4.

Leyba, I.M., Saraceno, M., Solman, S.A., 2017. Air-sea heat fluxes associated to mesoscale eddies in the Southwestern Atlantic Ocean and their dependence on different regional conditions. Climate Dynamics 49, 2491-2501. Doi 10.1007/s00382-016-3460-5.

Lentini, C.D.A., Campos, E.J.D., Podestá, G.G., 2000. The annual cycle of satellite derived sea surface temperature on the western South Atlantic shelf. Revista Brasileira de Oceanografia 48, 93-105. Doi: http://doi.org/10.1590/S141377392000000200001.

Lentini, C.A.D., Olson, D.B., Podesta, G.P., 2002. Statistics of Brazil Current rings observed from avhrr: 1993 to 1998. Geophysical Research Letters 29, 1-4. Doi: http://doi.org/10.1029/2002GL015221.

Liu, L., Xu, Z.X., Huang, J.X., 2012. Spatiotemporal variation and abrupt changes for major climate variables in the Taihu Basin, China. Stochastic Environmental Research Risk Assessment 26, 777-791. doi 10.1007/s00477011-0547-8.

Lumpkin, R., Garzoli S., 2011. Interannual to decadal changes in the western South 
Atlantic's surface circulation. Journal of Geophysical Research 116, C01014. Doi:10.1029/2010JC006285.

Mann, H.B., 1945. Non-parametric test against trend. Econometrika 13, 245-259.

Mata, M.M., Garcia, C.A.E., 2011. Oceano Austral e o clima, in: Goldenberg, J. (Org.), Antártica e as Mudanças Globais: um desafio para a humanidade. Editora Edgar Blücher Ltda, São Paulo, pp. 53-67.

Matano, R.P., Palma, E.D., Piola, A.R., 2010. The influence of the Brazil and Malvinas Currents on the southwestern Atlantic shelf circulation, Ocean Science Discussions 7, 1-35. Doi: 10.5194/osd-7-837-2010.

Mcbean, G., 2004. Climate change and extreme weather: a basis for action. Natural Hazards 31, 177-190.

Moreira, J.G.V., Naghettini, M., 2016. Detecção de Tendências Monotônicas Temporais e Relação com Erros dos Tipos I e II: Estudo de Caso em Séries de Precipitações Diárias Máximas Anuais do Estado do Acre. Revista Brasileira de Meteorologia 31, 394-402. http://doi.org/10.1590/0102778631231420140155.

Nobre, C.A., Reid, J., Veiga, A.P.S., 2012. Fundamentos científicos das mudanças climáticas, $1^{\text {a }}$ ed. - Rede Clima/INPE São José dos Campos.

Oliveira, M.M.F. de, Ebecken, N.F.F., Oliveira, J.L.F. de, Gilleland, E., 2011. Generalized extreme wind speed distributions in South America over the Atlantic Ocean region. Theoretical and Applied Climatology 104, 377385. Doi: 10.1007/s00704-010-0350-3.

Olson D., Podestá G.P., Brown O.B., 1988. Temporal variations in the separation of Brazil and Malvinas currents. Deep Sea Research 35, 1971-1980. Doi: http://doi.org/10.1016/01980149(88)90120-3.

Paniagua, G.F., Saraceno, M., Piola, A.R., Guerrero, R.A., Provost, C., Ferrari, R., Lago, L.S., Artana, C.I., 2018. Malvinas Current at 408S-418S: First Assessment of Temperature and Salinity Temporal Variability. Journal of Geophysical Research: Oceans 123, 5323-5340. Doi:10.1029/2017JC013666.

Peterson, R.G., Stramma, L., 1991. Upper-level circulation in the south atlantic ocean. Progress in Oceanography 26, 1-73.

Pettitt, A.N., 1979. A non-parametric approach to change point problem. Applied Statistics 28, 126-135.
Pezzi, L.P., Souza, R.B., Dourado, M.S., Garcia, C.A.E., Mata, M.M., Silva-Dias M. A. F., 2005. Ocean-atmosphere in situ observations at the Brazil-Malvinas Confluence region. Geophysical Research Letters 32, L22603. Doi: http://repositorio.furg.br/handle/1/1903

Pezzi, L.P., de Souza, R.B., Quadro, M.F.L., 2016. Uma Revisão dos Processos de Interação Oceano-Atmosfera em Regiões de Intenso Gradiente Termal do Oceano Atlântico Sul baseada em Dados Observacionais. Revista Brasileira de Meteorologia 31, 428-453. Doi: http://doi.org/10.1590/0102778631231420150032.

Piola, A.R., Avellaneda, N.M., Guerrero, R.A., Jardón, F.P., Palma, E. D., Romero, S.I., 2009. Malvinas-slope water intrusions on the northern Patagonia continental shelf. Ocean Science. Discussions 6, 2939-2974. Doi: 10.5194/os-6345-2010.

Piola, A.R., Palma, E. D., Bianchi, A.A., Castro, B.M., Dottori, M., Guerrero, R.A., Marrari, M., Matano, R.P., Möller Jr, O.O., Saraceno, M., 2018. Physical Oceanography of the SW Atlantic Shelf: A Review, in: Hoffmeyer, M., Sabati, M.E., Brandini, F.P.,Calliari, D.L., Santinelli, N.H. (Eds), Plankton Ecology of the Southwestern Atlantic From the Subtropical to the Subantarctic Realm. Springer, Cham, pp. 574.

Podestá, G.P., Brown, O.B., Evans, R.H., 1991. The Annual Cycle of Satellite-derived Sea Surface Temperature in the Southwestern Atlantic Ocean. Journal of Climate 4, 457-467. Doi: http://doi.org/10.1590/S141377392000000200001.

Rahman, M.A,, Yunsheng, L., Sultana, N., 2017. Analysis and prediction of rainfall trends over Bangladesh using Mann-Kendall, Spearman's rho tests and ARIMA model. Meteorology Atmospheric Physics 129(4):409-424. Doi: 10.1007/s00703-016-0479-4.

Rocha, F.P. da, Aravequia, J.A., Ribeiro, B.Z., 2016. Estudo de Ciclones e de Padrões de Circulação Atmosférica no Oceano Atlântico Sul Próximo à Costa das Regiões Sul e Sudeste do Brasil Usando Dados da Reanálise do EraInterim. Revista Brasileira de Meteorologia [online] 31, 141-156. Disponível: http://doi.org/10.1590/0102-778631220140151. Acesso: 01 set. 2020.

Ryan, D. 2015. From commitment to action: a literature review on climate policy 
implementation at city level. Climatic Change 131, 519-529. Doi 10.1007/s10584-015-1402-6

Sabatini, M., Reta, R., Matano, R., 2004. Circulation and zooplankton biomass distribution over the southern Patagonian shelf during late summer, Continental Shelf Research 24, 1359-1373. Doi: http://doi.org/10.1016/j.csr.2004.03.014.

Santos, C. A., Lima, A. M. M., Farias, M. H. C. S., Aires, U. R. V., Serrão, E. A. O., (2016). Análise estatística da não estacionariedade de séries temporais de vazão máxima anual diária na bacia hidrográfica do rio pardo. HOLOS 32, 179-193. Doi: 10.15628/holos.2016.4892

Sen, P.K., 1968. Estimates of the Regression Coefficient based on Kendall's Tau. Journal of the American Statistical Association 63, 13791389.

Silva, C.B., Silva, M.E.S., 2016. Análise espectral da temperatura da superfície do mar global no período de 1884 a 2014. Boletim Paulista de Geografia 95, 79-97.

Simões, J.C., 2011. O ambiente antártico: domínio de extremos, in: Goldenberg, J. (Org.), Antártica e as Mudanças Globais: um desafio para a humanidade. Editora Edgar Blücher Ltda, São Paulo, pp. 15-27.

Smith, P.J., 2000. The importance of the horizontal distribution of heating during extratropical cyclone development. Monthly Weather Review 128, 3692-3694. Doi: 10.1175/15200493(2000)128<3692:TIOTHD>2.0.CO;2.

Soppa, M.A., Souza, R.B., Pezzi, L.P., 2011. Variabilidade das Anomalias de Temperatura da Superfície do Mar no Oceano Atlântico Sudoeste e sua Relação com o Fenomeno el Niño-Oscilação Sul. Revista Brasileira de Meteorologia.26, 375-391. Doi: https//www.researchgate.net/publication/31303 8745

Spearman, C., 1904. The proof and measurement of associationbetween two things. American Journal of Psychology 15, 72-10.

Stramma, L., Peterson, R.G., 1990. The South Atlantic Current. Journal of Physical Oceanography 20, 846-859.

Tao, H., Fraederich, K., Menz, C., Zhai, J., 2014. Trends in extreme temperature indices in the Poyang Lake Basin, China. Stochastic Environmental Research and Risk Assessment 28, 1543-1553. Tomaziello, A.C.N., Gandu, A.W., 2013. Impacto da temperatura da superfície do mar na simulação da Zona de Convergência do Atlântico Sul. Revista Brasileira de Meteorologia 28, 291-304. Doi: http://doi.org/10.1590/S010277862013000300006.

Turner, J., Colwell, S.R., Marshall, G.J., LachlanCope, T.A., Andrew, M.C., Jones, P.D., Lagun, V., Reid, P.A., Iagovkina, S,. 2005. Antarctic climate change during the last 50 years. Journal of Climatology 25, 279-294. Doi: http://doi.org/10.1002/joc.1130.

Turner, J., Lu, H., White, I., King, J. C., Phillips, T., Scott Hosking, J., Bracegirdle, T.J., Marshall, G.J., Mulvaney, R., Deb, P., 2016. Absence of 21st century warming on Antarctic Peninsula consistent with natural variability. Nature 535, 411-415.

Van Loon, A.F., Stahl, K., Di Baldassarre, G., Clark, J., Rangecroft, S., Wanders, N., Gleeson, T., Van Dijk, A.I., Tallaksen, L.M., Hannaford, J., 2016. Drought in a human-modified world: reframing drought definitions, understanding, and analysis approaches. Hydrology and Earth System Sciences 20, 3631-3650. Doi: http://doi.org/10.5194/hess-20-3631-2016.

Vera, C.S., Vigliarolo, P.K., Berbery, E.H., 2002. Cold season synoptic scale waves over subtropical South America. Monthly Weather Review 130, 684-699. doi.org/10.1175/15200493(2002)130<0684:CSSSWO>2.0.CO;2.

WMO. WORLD METEOROLOGICAL ORGANIZATION, 2016. The Global Climate in 2011-2015. Genebra.

Xie, S.P., 2020. Ocean Warming Pattern Effect On Global And Regional Climate Change. AGU Advances, 1, ,e2019AV000130. Doi: http://doi.org/10.1029/2019AV000130.

Young, I.R., Ribal, A., 2019. Multiplatform evaluation of global trends in wind speed and wave height. Science 364, 548-552. Doi: 10.1126/science.aav9527.

Yue, S., Pilon, P., Cavadias, G., 2002. Power of the Mann-Kendall and Spearman's rho tests for detecting monotonic trends in hydrological series. Journal of Hydrology 259, 254-271. Doi: http://doi.org/10.1016/S0022-1694(01)00594-7.

Zavialov, P.O., Wainer, I., Absy, J.M., 1999. Sea surface temperature variability off southern Brazil and Uruguay as revealed from historical data since 1854. Journal of Geophysical Research 104, 21,021-21,032. Doi: 10.1029/1998JC900096. 